\title{
Leveraging Secondary Reflections and Mitigating Interference in Multi-IRS/RIS Aided Wireless Network
}

This paper was downloaded from TechRxiv (https://www.techrxiv.org).

\section{LICENSE}

CC BY-NC-SA 4.0

SUBMISSION DATE / POSTED DATE

08-12-2021 / 10-12-2021

\section{CITATION}

Nguyen, Tu; Nguyen, Diep; Renzo, Marco Di; Zhang, Rui (2021): Leveraging Secondary Reflections and Mitigating Interference in Multi-IRS/RIS Aided Wireless Network. TechRxiv. Preprint.

https://doi.org/10.36227/techrxiv.17140004.v1

$\mathrm{DOI}$

10.36227/techrxiv.17140004.v1 


\title{
Leveraging Secondary Reflections and Mitigating Interference in Multi-IRS/RIS Aided Wireless Network
}

\author{
Tu V. Nguyen, Member, IEEE, Diep N. Nguyen, Senior Member, IEEE, \\ Marco Di Renzo, Fellow, IEEE, and Rui Zhang, Fellow, IEEE
}

\begin{abstract}
Reconfigurable surfaces (RS) have recently emerged as an enabler for smart radio environments where they are used to actively tailor/control the radio propagation (e.g., to support users under adverse channel conditions). If multiple RSs are deployed (e.g., coated on various buildings) to support different groups of users, it is critical to jointly optimize the phase-shifts of all RSs to mitigate their interference as well as to leverage the secondary reflections amongst them. Motivated by the above, this paper considers the uplink transmissions of multiple users that are grouped and supported by multiple RSs to communicate with a multi-antenna base station (BS). We first formulate two optimization problems: the weighted sumrate maximization and the minimum achievable rate (from all users) maximization. Unlike existing works that considered single user or single RS or multiple RSs without inter-RS reflections, the considered problems require one to optimize the phase-shifts of all RSs' elements and all beamformers at the multi-antenna BS. The two problems turn out to be non-convex and thus are difficult to be solved in general. Moreover, the inter-RS reflections give rise to the coupling of the phase-shifts amongst RSs, making the optimization problems even more challenging to solve. To tackle them, we design alternating optimization algorithms that provably converge to locally optimal solutions. Simulation results reveal that by properly managing interference and leveraging the secondary reflections amongst RSs, there is a great benefit of deploying more RSs to support different groups of users and so as to achieve a higher rate per user. This gain is even more significant with a larger number of elements per RS. In contrast, without properly managing the secondary reflections, increasing the number of RSs can adversely impact the network throughput, especially for higher transmit power.
\end{abstract}

Index Terms-Intelligent reflecting surface (IRS), reconfigurable intelligent surface (RIS), secondary reflections, multiIRS/RIS interference, multi-user communications, cooperative beamforming.

\section{INTRODUCTION}

Reconfigurable surface (RS) also known as Intelligent Reflecting Surface (IRS) or Reconfigurable Intelligent Surface (RIS) has been emerging as an enabler for realizing so-called

Tu V. Nguyen is with the College of Engineering and Computer Science, VinUniversity, Ha Noi, Viet Nam (e-mail: tu.nv@ vinuni.edu.vn).

Diep N. Nguyen is with the School of Electrical and Data Engineering, University of Technology Sydney, Sydney, NSW 2007, Australia (e-mail: diep.nguyen@uts.edu.au).

M. Di Renzo is with Université Paris-Saclay, CNRS, CentraleSupélec, Laboratoire des Signaux et Systèmes, 3 Rue Joliot-Curie, 91192 Gif-surYvette, France. (marco.di-renzo@universite-paris-saclay.fr).

Rui Zhang is with the Department of Electrical and Computer Engineering, National University of Singapore, Singapore 117583 (e-mail: elezhangg@nus.edu.sg).

This work was supported in part by the VinUniversity Seed Grant program. smart radio environments in which the radio propagation can be deliberately controlled/tailored by deploying nearly-passive dynamic metasurfaces [1]-[5]. A wireless system assisted by RSs hence can "reconfigure" the environment to combat the shadowing and fading impairments or to create well-scattered environment for spatial multiplexing. An RS is a planar metasurface that is made up of a large number of passive reflecting elements. Each of these elements can be optimized to alter the amplitude and/or the phase-shift of the reflected signal. These elements together can help effectively "reshape" the wireless channels [1]-[5]. Such an ability to reconfigure the radio medium provides another new dimension of freedom to design wireless systems. Note that all conventional wireless optimization/designs take channel/radio environment as an input to adapt with, instead of actively "tailoring/optimizing" it [6].

With multiple RSs being deployed (e.g., coated on various buildings) to support distributed users, it is critical to jointly optimize the phase-shifts and/or amplitudes of all RSs to mitigate the interference as well as to leverage the secondary reflections amongst RSs. For example, consider the uplink transmission from multiple users that are grouped and supported by multiple RSs to a multi-antenna base station (BS), as shown in Fig. 1. Due to the close proximity amongst these groups of users, the RSs can be deployed close to each other. The signal from a given user not only reaches the BS by reflecting onto the dedicated RS for the user's group (referred to as the primary reflection) but also can traverse to and reflect on nearby RSs before reaching the BS (referred to as the secondary reflection or multi-RS reflection). It is clear that under such a scenario, besides the primary reflections from users to the BS via each RS, the secondary reflections amongst multiple RSs cannot be ignored in general. These secondary reflections can either cause multi-user and/or multi-RS interference or strengthen the signal reception at the BS. Therefore, by jointly tuning the phase-shifts of all RSs, one can simultaneously manage the interference and leverage the multi-RS "secondary" reflections to enhance the signal reception at the BS. Such an observation is similar to multi-cell cooperative multi-input multi-output (MIMO) communications [36].

Most existing works on multiple RSs, e.g., [13]-[15], ignored the secondary reflections amongst RSs. In [13], the authors studied a resource allocation problem for a downlink wireless communication network with multiple distributed RSs 
to maximize the system's energy efficiency with a maximum transmit power constraint and minimum rate requirements. The authors of [14] aimed to maximize the minimum signal-tointerference-plus-noise ratio (SINR) amongst users by jointly optimizing the transmit precoding vector at the BS and the phase-shifts at the RSs for a downlink multi-RS system. In [15], the outage probability and the average sum-rate for a single source, single destination and multi-RS system were studied where the best RS is selected to be active at a time. Like many other works that ignored the secondary reflections by justifying that the strength of signal reflected onto multiple RSs is negligible, such an assumption is only reasonable when the RSs are far from each other or the signal is reflected on many RSs (e.g., more than two). For the case when the RSs are deployed close to each other, e.g., in central business districts (CBDs) or dense deployment scenarios, as shown in this paper, the secondary reflections are in fact significant. Additionally, all aforementioned works did not study the interference management nor leverage the cooperation amongst RSs via "secondary" reflections. In [16], [17], the authors considered the beamforming design for the case with two RSs in which one is placed close to the BS and the other is placed close to the users. The authors show that there is a significant gain as compared to the case with a single RS. However, in the considered scenario there were only two RSs that aim to support a single group of users. Therefore, the problems in [16], [17] do not account for the interference amongst RSs and amongst different groups of users. In practice, especially in a dense urban environment where shadowing and fading are severe (e.g., caused by multiple buildings/structures), multiple RSs can be deployed within one cell to support various groups of users whose direct links to the BS are weak or blocked.

Given the above, this work takes the first step in exploring the impact of secondary reflections among multiple RSs by considering the uplink transmission of multiple groups of users that are supported by multiple RSs to communicate with a multi-antenna BS as illustrated in Fig. 1 [18]. For this scenario, we consider two commonly-adopted optimization problems: the weighted sum-rate maximization and the minimum-SINR maximization [18] in which we jointly optimize not only the phase-shifts of all RSs but also the beamforming vectors at the BS for all users. As aforementioned, besides the multi-user interference from multiple groups of users, multi-RS reflection also induces additional multi-user interference. For that the two problems are shown to be non-convex. The problems actually become more challenging to solve due to the coupling of the phase-shifts amongst RSs. Specifically, the adjustment of the phase-shifts at one RS also impacts the reflected signals from other RSs. As such, optimizing/tuning the phase-shifts at one RS [7]-[12] or multiple RSs but for a single group of users (e.g., [13]-[17], [38]-[49]) is insufficient in general. In fact, we need to optimize the RS elements for all RSs and all users while considering the coupling among them due to the inter-RS reflections.

Maximizing the weighted sum-rate for multi-user MIMO systems without RS has been well studied, for example, in [29]-[33]. Some recent works also studied the single or multiple RSs considering the weighted sum-rate such as [37]-
[45]. In [37], the authors considered the downlink of a multiuser multi-input single-output (MISO) system with a single RS and jointly optimized the transmit beamforming and phaseshifts of RS's elements. In [38], the authors used multiple RSs deployed at the cell-edge to improve the signal reception by optimizing the precoding matrices at the BS and the phaseshifts of the RSs. The scenarios with multiple BSs, multiple RSs, and multiple users in downlink communications were studied in [39] where the RS-user association design was optimized considering the co-channel interference. In [46][49], the authors considered the multi-beam multi-hop routing problem for a downlink of a multi-RS and multi-user system in which each user communicates with the BS with the help of a set of RSs. Note that in these works, even though there are multiple RSs involved in communications from the BS to each user, only RSs' primary reflections were considered and the signal is successively reflected on a sequence of RSs. For the minimum-SINR maximization problem, the work in [16], [17] is a special case of the problem investigated in our work by setting the number of the RSs to two and assign no user to the second RS. To the best of our knowledge, none of the existing works in the literature has considered the secondary reflections for multiple RSs in general. Our major contributions in this paper are summarized as follows:

- First, we study the impact of the inter-RS or secondary reflections in a multi-user, multi-RS network. To this end, we formulate and solve two well-known optimization problems: the weighted sum-rate maximization and the minimum-SINR maximization over the users.

- Second, we tackle these two non-convex optimization problems by designing alternating optimization algorithms that are proved to converge to locally optimal solutions. For the first problem, we transform each subproblem to a difference of convex (DC) structure and use an available software CVXPY/DCCP package in Python [26], [27] to efficiently solve it. While for the second problem, a relaxed version of each of its sub-problems is transformed to a semidefinite program (SDP) problem which can be efficiently solved by SDP standard solvers. Note that unlike existing works that did not account for the inter-RS reflections, the two considered problems are more challenging as we need to deal with the coupling of phase-shifts among different RSs' elements.

- Third, by optimizing the phase-shifts of all RSs as well as the beamforming vectors for all users at the BS, we show that the inter-RS reflections can also be leveraged to contribute to the signal reception at the $\mathrm{BS}$, leading to an improved rate per user. We observe that the gain is more significant with a larger number of elements per RS.

- Last, we perform extensive simulations to evaluate the impact of secondary reflections in various settings. For example, we observe about $6.4 \%, 15.6 \%$, or $29.9 \%$ rate gains when exploiting the secondary reflections for a system with 2 , 4, or $6 \mathrm{RSs}$, respectively. Also, when the number of RS elements is increased, the gain is also increased, for instance, there is about $7.2 \%, 15.6 \%$, and 
$27.9 \%$ gains for a system with four RSs each with 32, 64 and 128 elementss, respectively. However, without properly managing the secondary reflections, increasing the number of RSs may adversely impact the network throughput, especially for high transmit power (e.g. the achievable rate of six RSs is worse than that of four RSs at transmit power of $25.5 \mathrm{dBm}$ or higher).

The rest of this paper is organized as follows. In Section II, the system model and the problem formulation for multi-RS system are presented. In Section III, a locally optimal solution for the weighted sum-rate maximization is presented. The minrate/SINR maximization problem is studied in Section IV. In Section V, numerical results are discussed, and conclusions are drawn in Section VI.

\section{System Model}

We consider a system with an $N$-antenna BS and $K$ singleantenna users. Due to the presence of blocking objects in the network, users are partitioned/clustered into $L$ groups whose direct radio links to the BS are not available. Such a scenario is often the case in dense CBD/urban areas with high-rise buildings and structures. $L$ RSs are then used to support the transmission of these users, as depicted in Fig. 1 . We assume that the $l$ th $\mathrm{RS}$ is comprised of $M_{l}$ elements and serves $K_{l}$ single-antenna users in the $l$-th group $l=1,2, \cdots, L$. We note that $\sum_{l=1}^{L} K_{l}=K$ is the total number of users. To simplify the notation, we denote the set of users in the $l$ th group, RS elements of the $l$ th RS, and RSs as $\mathcal{K}_{l}=\left\{1,2, \cdots, K_{l}\right\}, \mathcal{M}_{l}=\left\{1,2, \cdots, M_{l}\right\}$, and $\mathcal{L}=\{1,2, \cdots, L\}$, respectively.

It is worth noting that in general, each RS element can be controlled to adjust both its amplitude and/or phase. However, similar to many other works in literature (e.g., [1], [3], [4], [16], [17]), for simplicity and cost-effective implementation, the amplitude of all RS elements is set to one and we only tune/optimize their phases. The results and analysis in the sequel can be easily extended to optimize both amplitudes and phases.

The signal from the $k$-th user in the $l$-th group (denoted as the $k^{(l)}$-th user for short) can reach the BS by one of the following paths via at least one RS: (i) single or primary reflection from the $k^{(l)}$-th user onto any $\tilde{l}$-th $\mathrm{RS}$ then to the BS, (ii) double reflections (via two RSs) from $k^{(l)}$-th user to the $l$-th RS, then to the $\tilde{l}$-th RS before reaching the BS (where $l \neq \tilde{l}$ ), (iii) triple or more reflections (via three or more RSs), e.g., from $k^{(l)}$-th user to the $l$-th RS, then to the $\tilde{l}$ th RS to the $l^{\prime}$-th RS to the BS (where $l, \tilde{l}$ and $l^{\prime}$ are pair-wise different). As aforementioned, most of the works on multiple RSs in the literature, e.g., [13]-[17], [38]-[49], only consider the primary reflection (type (i)) but not the secondary nor the triple/more reflections amongst RSs. Unlike the triple or more reflections (type (iii)) during which a signal traverses through more than two RSs hence can be negligible due to much larger effective pathloss, the secondary reflections amongst RSs may not be negligible in practice, and this is the focus of the present paper. On the one hand, these secondary reflections can cause unwanted interference at the BS. On the other hand, if we

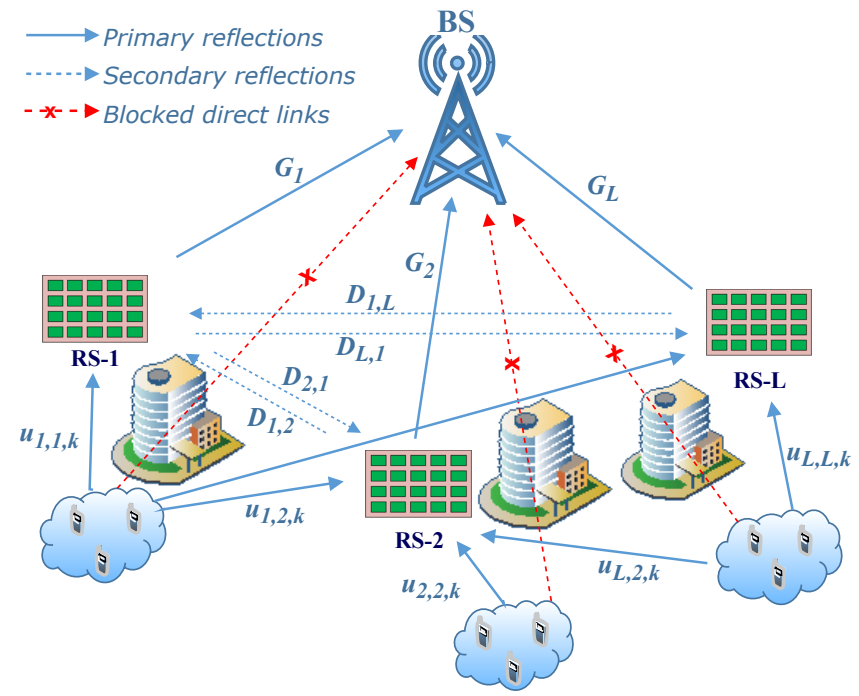

Fig. 1: Multi-RS assisted multi-groups MIMO communication system.

can properly manage such interference and leverage them, these secondary reflections can constructively contribute to the signal detection/decoding at the BS. Note that the secondary reflections are particularly significant when the RSs are not too far from each other, e.g., in urban environments with dense deployment of the RSs.

Let $\boldsymbol{u}_{\tilde{l}, k^{(l)}} \in \mathbb{C}^{M_{\tilde{l}} \times 1}, \boldsymbol{D}_{l, \tilde{l}} \in \mathbb{C}^{M_{l} \times M_{\tilde{l}}}$, and $\boldsymbol{G}_{l} \in \mathbb{C}^{N \times M_{l}}$ denote the baseband equivalent channel matrix from the user $k$ in the group $l$ to the RS- $\tilde{l}$, the baseband equivalent channel matrix from the RS- $\tilde{l}$ to the RS-l (for $\tilde{l} \neq l$ ), and the baseband equivalent channel matrix from RS-l to the BS links, respectively, with $l, \tilde{l} \in \mathcal{L}$ and $k \in \mathcal{K}_{l}$. Let $\boldsymbol{\theta}_{l} \in \mathbb{C}^{M_{l} \times 1}$ denote the phase-shifts vector of the RS-l. We assume the direct links from users to the BS are weak and can be ignored. Thus, the effective channel from the $k^{(l)}$-th user can be written as follows

$$
\boldsymbol{h}_{k^{(l)}}=\sum_{\tilde{l}=1}^{L} \boldsymbol{G}_{\tilde{l}} \boldsymbol{\Phi}_{\tilde{l}} \boldsymbol{u}_{\tilde{l}, k^{(l)}}+\sum_{\substack{\tilde{i}=1 \\ l \neq l}}^{L} \boldsymbol{G}_{\tilde{l}} \boldsymbol{\Phi}_{\tilde{l}} \boldsymbol{D}_{\tilde{l}, l} \boldsymbol{\Phi}_{l} \boldsymbol{u}_{l, k^{(l)}}
$$

for $l \in \mathcal{L}$, and $k^{(l)} \in \mathcal{K}_{l}$, where $\boldsymbol{\Phi}_{l}=\operatorname{diag}\left(\boldsymbol{\theta}_{l}\right)$ denotes the diagonal reflection matrix of RS-l.

Denote $\boldsymbol{R}_{\tilde{l}, k^{(l)}}=\boldsymbol{G}_{\tilde{l}} \operatorname{diag}\left(\boldsymbol{u}_{\tilde{l}, k^{(l)}}\right) \in \mathbb{C}^{N \times M_{\tilde{l}}}$ as the cascaded channel matrix from the $k^{(l)}$-th user to the $\tilde{l}$-th RS to the BS (excluding the phase-shifts at the $\tilde{l}$-th RS) ${ }^{1}$. Also, denote $\tilde{\boldsymbol{D}}_{\tilde{l}, k^{(l)}} \equiv\left[\tilde{\boldsymbol{d}}_{\tilde{l}, k^{(l)}, 1}, \ldots, \tilde{\boldsymbol{d}}_{\tilde{l}, k^{(l)}, M_{l}}\right] \triangleq \boldsymbol{D}_{\tilde{l}, l} \operatorname{diag}\left(\boldsymbol{u}_{l, k^{(l)}}\right) \in$ $\mathbb{C}^{M_{i} \times M_{l}}$ as the cascaded channel from the $k^{(l)}$-th user to the $l$-th RS to the $\tilde{l}$-th RS (without the RS elements' phase-shift), where $\tilde{\boldsymbol{d}}_{\tilde{l}, k^{(l)}, j}$ is the $j$-th column vector of $\tilde{\boldsymbol{D}}_{\tilde{l}, k^{(l)}}$. Thus, we

\footnotetext{
${ }^{1}$ That is, the $(i, j)$-th entry of $\boldsymbol{R}_{\tilde{l}, k(l)}$ is the instantaneous channel gain from the $k^{(l)}$-th user to the $j$-th RS element (of the $\tilde{l}$-th RS) to the $i$-th receive antenna, in which the phase-shift of this RS element is set to zero.
} 
can rewrite (1) as follows

$$
\begin{aligned}
\boldsymbol{h}_{k^{(l)}} & =\sum_{\tilde{l}=1}^{L} \boldsymbol{R}_{\tilde{l}, k^{(l)}} \boldsymbol{\theta}_{\tilde{l}}+\sum_{\tilde{l}=1, \tilde{l} \neq l}^{L} \boldsymbol{G}_{\tilde{l}} \boldsymbol{\Phi}_{\tilde{l}} \tilde{\boldsymbol{D}}_{\tilde{l}, k^{(l)}} \boldsymbol{\theta}_{l} \\
& =\sum_{\tilde{l}=1}^{L} \boldsymbol{R}_{\tilde{l}, k^{(l)}} \boldsymbol{\theta}_{\tilde{l}}+\sum_{\tilde{l}=1, \tilde{l} \neq l}^{L} \sum_{j=1}^{M_{l}} \boldsymbol{G}_{\tilde{l}} \operatorname{diag}\left(\tilde{\boldsymbol{d}}_{\tilde{l}, k^{(l)}, j}\right) \boldsymbol{\theta}_{\tilde{l}} \theta_{l, j} \\
& =\sum_{\tilde{l}=1}^{L} \boldsymbol{R}_{\tilde{l}, k^{(l)}} \boldsymbol{\theta}_{\tilde{l}}+\sum_{\tilde{l}=1, \tilde{l} \neq l}^{L} \sum_{j=1}^{M_{l}} \boldsymbol{Q}_{\tilde{l}, k^{(l)}, j} \boldsymbol{\theta}_{\tilde{l}} \theta_{l, j},
\end{aligned}
$$

where $\boldsymbol{Q}_{\tilde{l}, k^{(l)}, j} \triangleq \boldsymbol{G}_{\tilde{l}} \operatorname{diag}\left(\tilde{\boldsymbol{d}}_{\tilde{l}, k^{(l)}, j}\right) \in \mathbb{C}^{N \times M_{\tilde{l}}}$ denotes the total cascaded channel matrix from the $k^{(l)}$-th user to the $j$-th element of the RS- $l$ to the RS- $\tilde{l}$ to the BS without the phaseshifts at the RS- $l$ and the RS- $\tilde{l}$, where $l \in \mathcal{L}, j \in \mathcal{M}_{l}$ and $k^{(l)} \in \mathcal{K}_{l}$. From (2), we can see that it suffices to estimate $\left\{\boldsymbol{R}_{\tilde{l}, k^{(l)}}\right\}$ and $\left\{\boldsymbol{Q}_{\tilde{l}, k^{(l)}, j}\right\}$ for jointly designing the passive beamforming coefficients $\left\{\boldsymbol{\theta}_{l}\right\}$ in the considered multi-RS cooperative system [16], [17], [21]. In this paper, we assume that all the cascaded channel matrices $\left\{\boldsymbol{R}_{\tilde{l}, k^{(l)}}\right\}$ and $\left\{\boldsymbol{Q}_{\tilde{l}, k^{(l)}, j}\right\}$ are accurately estimated at the BS.

During the uplink data transmission, the received signal at the $\mathrm{BS}$ is given by

$$
\begin{aligned}
\boldsymbol{y} & =\sum_{l=1}^{L} \sum_{k=1}^{K_{l}} \boldsymbol{h}_{k^{(l)}} s_{k^{(l)}}+\boldsymbol{n} \\
& =\sum_{l=1}^{L} \sum_{k=1}^{K_{l}}\left(\sum_{\tilde{l}=1}^{L} \boldsymbol{R}_{\tilde{l}, k^{(l)}} \boldsymbol{\theta}_{\tilde{l}}+\right. \\
& \left.\sum_{\substack{\tilde{l}=1 \\
\tilde{l} \neq l}}^{L} \sum_{j=1}^{M_{l}} \boldsymbol{Q}_{\tilde{l}, k^{(l)}, j} \boldsymbol{\theta}_{\tilde{l}} \theta_{l, j}\right) s_{k^{(l)}}+\boldsymbol{n},
\end{aligned}
$$

where $s_{k^{(l)}}$ is the $k^{(l)}$-th user's transmitted data symbol with the transmit power equal to $P_{k^{(l)}}$, and $\boldsymbol{n} \sim \mathcal{C N}\left(0, \sigma^{2} \boldsymbol{I}_{N}\right)$ is the additive white Gaussian noise (AWGN) vector at the BS with zero mean and covariance matrix $\sigma^{2} \boldsymbol{I}_{N}$ where $\sigma^{2}$ is the equivalent noise power $\left(\boldsymbol{n} \in \mathbb{C}^{N \times 1}\right.$ ) and $\boldsymbol{I}_{N}$ is the identity matrix of size $N$. At the BS, a linear receive beamforming vector $\boldsymbol{w}_{k^{(l)}} \in \mathbb{C}^{N \times 1}$ is applied to decode $s_{k^{(l)}}$, which results in

$$
\begin{aligned}
\tilde{y}_{k^{(l)}}= & \boldsymbol{w}_{k^{(l)}}^{H} \boldsymbol{y} \\
= & \boldsymbol{w}_{k^{(l)}}^{H} \sum_{\tilde{l}=1}^{L} \sum_{\tilde{k}=1}^{K_{l}}\left(\sum_{l^{\prime}=1}^{L} \boldsymbol{R}_{l^{\prime}, \tilde{k}^{(\tilde{l})}} \boldsymbol{\theta}_{l^{\prime}}+\right. \\
& \left.\sum_{l^{\prime} \neq \tilde{l}}^{L} \sum_{j=1}^{M_{\tilde{l}}} \boldsymbol{Q}_{l^{\prime}, \tilde{k}^{(\tilde{l})}, j} \boldsymbol{\theta}_{l^{\prime}} \theta_{\tilde{l}^{, j}}\right) s_{\tilde{k}^{(\tilde{l})}}+\boldsymbol{w}_{k^{(l)}}^{H} \boldsymbol{n} .
\end{aligned}
$$

$$
\gamma_{k^{(l)}}=\frac{P_{k^{(l)}}\left|\boldsymbol{w}_{k^{(l)}}^{H}\left(\sum_{\tilde{l}=1}^{L} \boldsymbol{R}_{\tilde{l}, k^{(l)}} \boldsymbol{\theta}_{\tilde{l}}+\sum_{\tilde{l} \neq l}^{L} \sum_{j=1}^{M_{l}} \boldsymbol{Q}_{\tilde{l}, k^{(l)}, j} \boldsymbol{\theta}_{\tilde{l}} \theta_{l, j}\right)\right|^{2}}{\sum_{(\tilde{l}, \tilde{k}) \neq(l, k)} P_{\tilde{k}^{(\tilde{l})}}\left|\boldsymbol{w}_{k^{(l)}}^{H}\left(\sum_{l^{\prime}=1}^{L} \boldsymbol{R}_{l^{\prime}, \tilde{k}^{(\tilde{l})}} \boldsymbol{\theta}_{l}+\sum_{l^{\prime} \neq \tilde{l}}^{L} \sum_{j=1}^{M_{\tilde{l}}} \boldsymbol{Q}_{l^{\prime}, \tilde{k}^{(\tilde{l})}, j} \boldsymbol{\theta}_{l^{\prime}} \theta_{\tilde{l}, j}\right)\right|^{2}+\sigma^{2} \boldsymbol{w}_{k^{(l)}}^{H} \boldsymbol{w}_{k^{(l)}}}
$$

Thus, the SINR for decoding the information from the $k^{(l)}$-th user is given in (5), which is shown at the bottom of this page.

Given the SINR in (5) for all $k^{(l)}$ users, we can jointly optimize phase-shift design $\left\{\boldsymbol{\theta}_{l}\right\}$ 's for all RSs and the receive beamforming vectors $\boldsymbol{w}_{k^{(l)}}$ at the BS for all users to simultaneously mitigate the RS interference and leverage the secondary reflections amongst RSs. To this end, we consider two wellknown optimization problems in the following two sections.

\section{Weighted Sum-RATE MAXimization}

Let's first consider the weighted sum-rate maximization problem subject to the minimum rate requirement per user. The problem is formally formulated as follows

$$
\begin{aligned}
& \text { (P1): } \max _{\left\{\boldsymbol{w}_{k^{(l)}}\right\},\left\{\boldsymbol{\theta}_{l}\right\}} \sum_{l=1}^{L} \sum_{k=1}^{M_{l}} \alpha_{k^{(l)}} \log _{2}\left(1+\gamma_{k^{(l)}}\right) \\
& \text { s.t. } \quad \gamma_{k^{(l)}} \geq 2^{R_{k^{(l)}} / B}-1, \forall l \in \mathcal{L}, k \in \mathcal{K}_{l}, \\
& \quad\left|\theta_{l, j}\right|=1, \quad \forall l \in \mathcal{L}, \quad j \in \mathcal{M}_{l},
\end{aligned}
$$

where $\gamma_{k^{(l)}}$ is given in (5), $\left\{\alpha_{k^{(l)}}\right\}$ are predefined non-negative weights with $\sum_{k^{(l)}} \alpha_{k^{(l)}}=1$, and $\left\{R_{k^{(l)}}\right\}$ are also predefined minimum rates requirement of each user $k^{(l)}$.

Beside the multi-user interference term in the SINR expression given in (5), the problem (P1) is non-convex due to the unity constraint on the phase-shift $\left|\theta_{l, j}\right|=1$. To tackle it, we propose an $\mathrm{AO}$ algorithm that can be proved to converge to a locally optimal solution of the problem (P1).

\section{A. Optimize $\left\{\boldsymbol{w}_{k^{(l)}}\right\}$ 's for Fixed $\left\{\boldsymbol{\theta}_{l}\right\}_{l=1}^{L}$}

For fixed $\left\{\boldsymbol{\theta}_{l}\right\}_{l=1}^{L}$, the effective channel of each user $\boldsymbol{h}_{k^{(l)}}$ in (2) is fixed and thus problem (P1) is reduced to $K$ subproblems, each of which is equivalent to maximizing the SINR of the $k^{(l)}$-th user as given in (5) and can be formulated as

$$
\text { (P2): } \max _{\boldsymbol{w}_{k^{(l)}}} \frac{P_{k^{(l)}} \boldsymbol{w}_{k^{(l)}}^{H} \boldsymbol{h}_{k^{(l)}} \boldsymbol{h}_{k^{(l)}}^{H} \boldsymbol{w}_{k^{(l)}}}{\boldsymbol{w}_{k^{(l)}}^{H}\left(\sum_{\substack{(\tilde{c}, \tilde{k}) \\ \neq(l, k)}} P_{\tilde{k}^{(\tilde{l})}} \boldsymbol{h}_{\tilde{k}^{(\tilde{l})}} \boldsymbol{h}_{\tilde{k}^{(\tilde{l})}}^{H}+\sigma^{2} \boldsymbol{I}\right) \boldsymbol{w}_{k^{(l)}}} .
$$

It can be shown that $(\mathrm{P} 2)$ is a convex optimization problem, and in fact it has a closed-form optimal solution [23]. Let's denote $\boldsymbol{H}=\left[\boldsymbol{H}_{1}, \ldots, \boldsymbol{H}_{L}\right] \in \mathbb{C}^{N \times K}$ and $\boldsymbol{W}=$ $\left[\boldsymbol{W}_{1}, \ldots, \boldsymbol{W}_{L}\right] \in \mathbb{C}^{N \times K}$, where $\boldsymbol{H}_{\boldsymbol{l}}=\left[\boldsymbol{h}_{l, 1}, \ldots, \boldsymbol{h}_{K_{l}}\right] \in$ $\mathbb{C}^{N \times K_{l}}$ and $\boldsymbol{W}_{\boldsymbol{l}}=\left[\boldsymbol{w}_{l, 1}, \ldots, \boldsymbol{w}_{K_{l}}\right] \in \mathbb{C}^{N \times K}$, denote the effective user-to-BS channel matrix and the receive beamforming matrix applied at the BS, respectively. The problem (P2) has the optimal minimum mean squared error (MMSE) solution as [23]

$$
\boldsymbol{W}_{M M S E}=\left(\boldsymbol{H} \boldsymbol{P} \boldsymbol{P} \boldsymbol{H}^{H}+\sigma^{2} \boldsymbol{I}\right)^{-1} \boldsymbol{H} \boldsymbol{P},
$$


where $\boldsymbol{P}=\operatorname{diag}\left(\left[\sqrt{P_{1,1}}, \sqrt{P_{1,2}}, \ldots, \sqrt{P_{k_{L}^{(l)}}}\right]\right)$ is a diagonal transmit power matrix for all $K$ users. In practice, the equivalent noise power, $\sigma^{2}$, needs to be estimated; the solution to the problem (P2) can often be simplified to a sub-optimal zero-forcing (ZF) solution where the noise term $\sigma^{2} \boldsymbol{I}$ in (8) is omitted and the matrix pseudo inverse is applied [23].

\section{B. Optimize $\left\{\boldsymbol{\theta}_{l}\right\}_{l=1}^{L}$ for Fixed $\left\{\boldsymbol{w}_{k^{(l)}}\right\}$ 's}

For fixed $\left\{\boldsymbol{w}_{k^{(l)}}\right\}$ 's, the problem (P1) in (6) can be reformulated as follows

$$
\begin{gathered}
\text { (P3): } \max _{\left\{\boldsymbol{\theta}_{l}\right\}} \sum_{l=1}^{L} \sum_{k=1}^{M_{l}} \alpha_{k^{(l)}} \log _{2}\left(1+\gamma_{k^{(l)}}\right) \\
\text { s.t. } \quad \gamma_{k^{(l)}} \geq 2^{R_{k}(l)} / B-1, \forall l \in \mathcal{L}, k \in \mathcal{K}_{l}, \\
\left|\theta_{l, j}\right|=1, \forall l \in \mathcal{L}, j \in \mathcal{M}_{l} .
\end{gathered}
$$

Similar to (P1), the problem (P3) in (9) is also non-convex. We note that because of the secondary reflections, the SINR of a given user now depends not only on the phase-shift vector of one RS but also on those of other RSs. This is captured by the coupling of the $L$ phase-shift vectors $\left\{\boldsymbol{\theta}_{l}\right\}_{l=1}^{L}$ in $\gamma_{k^{(l)}}$ for each user in (P3). Note that most, if not all, of the existing works that did not account for the secondary reflections do not need to deal with this coupling among the $L$ phase-shift vectors $\left\{\boldsymbol{\theta}_{l}\right\}_{l=1}^{L}$. To tackle it in our work, we design an alternating algorithm to solve the problem (P3) in which we first fix $\left\{\boldsymbol{\theta}_{l}\right\}_{l \neq \hat{l}}$, then solve for $\boldsymbol{\theta}_{\hat{l}}$, for each $\hat{l} \in \mathcal{L}$ in an iterative manner. Note that this sub-problem is still non-convex. To solve it, we observe that the objective function is a difference of two convex (DC) functions. We thus convert it into a DC optimization problem and use the disciplined convex-concave programming (DCCP) method for solving it. This sub-problem is solved later in Section III.C.

In the following, we first derive $\gamma_{k^{(l)}}$ as a function of $\boldsymbol{\theta}_{\hat{l}}$. There are two scenarios to be considered: i) the $k^{(l)}$-th user belongs to the $\hat{l}$-th RS (i.e., $l=\hat{l}$ ) and ii) the $k^{(l)}$-th user does not belong to the $\hat{l}$-th $\mathrm{RS}$ (i.e., $l \neq \hat{l}$ ).

Theorem 1. The SINR of the $k^{(l)}$-th user at the BS can be written as a function of $\boldsymbol{\theta}_{\hat{l}}$ as follow.

$$
\gamma_{k^{(l)}}=\frac{\left|\boldsymbol{q}_{\hat{l}, k^{(l)}}^{H} \boldsymbol{\theta}_{\hat{l}}+p_{\hat{l}, k^{(l)}}\right|^{2}}{\sum_{\substack{(\tilde{l}, \tilde{k}) \\ \neq(l, k)}}\left|\boldsymbol{q}_{\hat{l}, \tilde{k}^{(\tilde{l})}}^{H} \boldsymbol{\theta}_{\hat{l}}+p_{\hat{l}, \tilde{k}^{(\tilde{l})}}\right|^{2}+\sigma_{k^{(l)}}^{2}}
$$

where

$$
\begin{gathered}
\sigma_{k^{(l)}}^{2} \triangleq \sigma^{2} \boldsymbol{w}_{k^{(l)}}^{H} \boldsymbol{w}_{k^{(l)}}, \\
\boldsymbol{q}_{\hat{l}, k^{(l)}} \triangleq\left\{\begin{array}{l}
\sqrt{P_{k^{(l)}}} \boldsymbol{w}_{k^{(l)}}^{H}\left(\boldsymbol{R}_{\hat{l}, k^{(l)}}+\boldsymbol{S}_{\hat{l}, k^{(l)}}\right) \\
\sqrt{P_{k^{(l)}}} \boldsymbol{w}_{k^{(l)}}^{H}\left(\boldsymbol{R}_{\hat{l}, k^{(l)}}+\boldsymbol{T}_{\hat{l}^{(l)}}\right) \\
\text { for } l=\hat{l} \\
\text { for } l \neq \hat{l}
\end{array}\right.
\end{gathered}
$$

and

$$
p_{\hat{l}, k^{(l)}} \triangleq \begin{cases}\sqrt{P_{k^{(l)}}} \boldsymbol{w}_{k^{(l)}}^{H} \boldsymbol{U}_{\hat{l}, k^{(l)}} & \text { for } l=\hat{l} \\ \sqrt{P_{k^{(l)}}} \boldsymbol{w}_{k^{(l)}}^{H}\left(\boldsymbol{U}_{\hat{l}, k^{(l)}}+\boldsymbol{S}_{\hat{l}^{(} k^{(l)}} \boldsymbol{\theta}_{l}\right) & \text { for } l \neq \hat{l}\end{cases}
$$

with $\boldsymbol{S}_{\hat{l}_{, k^{(l)}}}, \boldsymbol{T}_{\hat{l}, k^{(l)}}$ and $\boldsymbol{U}_{\hat{l}, k^{(l)}}$ being independent of $\boldsymbol{\theta}_{\hat{l}}$ as defined in (29) and (30), respectively.

Proof: See Appendix A.

We note that (10) has the same structure for all $k^{(l)}$ but the underlying terms are different for users that belong to the $\hat{l}$-th RS and those that do not, as shown in (12) and (13).

\section{Optimize $\boldsymbol{\theta}_{\hat{l}}$ for Fixed $\left\{\boldsymbol{\theta}_{\tilde{l}}\right\}_{\tilde{l} \neq \hat{l}}$ and Fixed $\left\{\boldsymbol{w}_{k^{(l)}}\right\}$ 's}

For each $\hat{l}$, we substitute $\gamma_{k^{(l)}}=\gamma_{k^{(l)}}\left(\boldsymbol{\theta}_{\hat{l}}\right)$ as a function of $\boldsymbol{\theta}_{\hat{l}}$ as shown in (10); then problem (P3) becomes

$$
\begin{gathered}
\text { (P3.1): } \max _{\boldsymbol{\theta}_{\hat{l}}} \sum_{l=1}^{L} \sum_{k=1}^{M_{l}} \alpha_{k^{(l)}} \log _{2}\left(1+\gamma_{k^{(l)}}\left(\boldsymbol{\theta}_{\hat{l}}\right)\right) \\
\text { s.t. } \quad \gamma_{k^{(l)}}\left(\boldsymbol{\theta}_{\hat{l}}\right) \geq 2^{R_{k^{(l)}} / B}-1, \forall l, k, \\
\quad\left|\theta_{\hat{l}, j}\right|=1, \forall j \in \mathcal{M}_{\hat{l}} .
\end{gathered}
$$

Due to its unity constraints on $\left\{\theta_{\hat{l}, j}\right\}$, the above problem is non-convex. However, we observe that $\gamma_{k^{(l)}}\left(\boldsymbol{\theta}_{\hat{l}}\right)$ is a ratio of two convex functions of $\boldsymbol{\theta}_{\hat{l}}$ (as shown in (10)). Hence, we can rewrite it as the difference of two convex functions. Consequently, to solve (P3.1), we first convert the problem to a DC optimization problem and use the DCCP method, for example, the CVXPY/DCCP package in Python [26], [27] to efficiently solve for it. Since the current CVXPY/DCCP solvers have limited support for complex variables, we need to convert our problem to real-valued variables. First, let's define

$$
\begin{aligned}
& \boldsymbol{Q}_{\hat{l}, k(l)} \triangleq\left[\begin{array}{cc}
\boldsymbol{q}_{\hat{l}^{(k)}}^{(r)} & -\boldsymbol{q}_{\hat{l}, k(l)}^{(i)} \\
\boldsymbol{q}_{\hat{l}, k(l)}^{(i)} & \boldsymbol{q}_{\hat{l}, k(l)}^{(r)}
\end{array}\right], \\
& \tilde{\boldsymbol{\theta}}_{\hat{l}} \triangleq\left[\begin{array}{c}
\boldsymbol{\theta}_{\hat{l}}^{(r)} \\
\boldsymbol{\theta}_{\hat{l}}^{(i)}
\end{array}\right], \\
& \tilde{\boldsymbol{p}}_{\hat{l}, k^{(l)}} \triangleq\left[\begin{array}{c}
p_{\hat{l}, k(l)}^{(r)} \\
p_{\hat{l}, k^{(l)}}^{(i)}
\end{array}\right],
\end{aligned}
$$

where $\boldsymbol{x}^{(r)}$ and $\boldsymbol{x}^{(i)}$ denote the real and imaginary part of a complex vector $\boldsymbol{x}$, respectively. Note that $\boldsymbol{Q}_{\hat{l}, k^{(l)}} \in$ $\mathbb{R}^{2 M_{\hat{\imath}} \times 2}, \tilde{\boldsymbol{\theta}}_{\hat{l}} \in \mathbb{R}^{2 M_{\hat{\imath}} \times 1}$, and $\tilde{\boldsymbol{p}}_{\hat{l}, k^{(l)}} \in \mathbb{R}^{2 \times 1}$. With the above definitions, we can rewrite

$$
\begin{aligned}
\left|\begin{array}{l}
\mid \boldsymbol{q}_{\hat{l}, k(l)}^{H} \\
\boldsymbol{\theta}_{\hat{l}}+p_{\hat{l}, k^{(l)}}
\end{array}\right|^{2} & \boldsymbol{\theta}_{\hat{l}}^{H} \boldsymbol{p}_{\hat{l}, k^{(l)}} \boldsymbol{p}_{\hat{l}, k^{(l)}}^{H} \boldsymbol{\theta}_{\hat{l}}+2 \Re\left\{p_{\hat{l}, k^{(l)}}^{H} \boldsymbol{p}_{\hat{l}, k^{(l)}}^{H} \boldsymbol{\theta}_{\hat{l}}\right\}+\left|\boldsymbol{p}_{\hat{l}, k^{(l)}}\right|^{2} \\
= & \tilde{\boldsymbol{\theta}}_{\hat{l}}^{T} \boldsymbol{Q}_{\hat{l}, k^{(l)}} \boldsymbol{Q}_{\hat{l}, k^{(l)}}^{T} \tilde{\boldsymbol{\theta}}_{\hat{l}}+2 \tilde{\boldsymbol{p}}_{\hat{l}, k^{(l)}}^{T} \boldsymbol{Q}_{\hat{l}, k^{(l)}}^{T} \tilde{\boldsymbol{\theta}}_{\hat{l}}+\left\|\tilde{\boldsymbol{p}}_{\hat{l}, k^{(l)}}\right\|_{2}^{2},
\end{aligned}
$$

where $\|\boldsymbol{x}\|_{2}$ denotes the $l_{2}$ norm of a vector $\boldsymbol{x}$. To further simplify the notation, we define

$$
\begin{gathered}
\boldsymbol{Z}_{\hat{l}, k^{(l)}} \triangleq \boldsymbol{Q}_{\hat{l}, k^{(l)}} \boldsymbol{Q}_{\hat{l}, k^{(l)}}^{T}, \quad \hat{\boldsymbol{Z}}_{\hat{l}_{, k^{(l)}}} \triangleq \sum_{(\hat{l}, \hat{k}) \neq\left(k^{(l)}\right)} \boldsymbol{Z}_{\hat{l}, k^{(l)}}, \\
\boldsymbol{z}_{\hat{l}, k^{(l)}} \triangleq \boldsymbol{Q}_{\hat{l}, k^{(l)}} \boldsymbol{Q}_{\hat{l}, k^{(l)}}^{T}, \quad \hat{\boldsymbol{z}}_{\hat{l}, k^{(l)}} \triangleq \sum_{(\hat{l}, \hat{k}) \neq\left(k^{(l)}\right)} \boldsymbol{z}_{\hat{l}, k^{(l)}},
\end{gathered}
$$


and

$$
c_{\hat{l}, k^{(l)}} \triangleq\left\|\tilde{\boldsymbol{p}}_{\hat{l}, k^{(l)}}\right\|_{2}^{2}, \quad \hat{c}_{\hat{l}, k^{(l)}} \triangleq \sigma_{k^{(l)}}^{2}+\sum_{(\hat{l}, \hat{k}) \neq\left(k^{(l)}\right)} v_{\hat{l}, k^{(l)}} .
$$

We then can rewrite $\gamma_{k^{(l)}}$ in (14) as a function of $\tilde{\boldsymbol{\theta}}_{\hat{l}}$ as follows

$$
\gamma_{k^{(l)}}=\gamma_{k^{(l)}}\left(\tilde{\boldsymbol{\theta}}_{\hat{l}}\right)=\frac{\tilde{\boldsymbol{\theta}}_{\hat{l}} \boldsymbol{Z}_{\hat{l}, k^{(l)}} \tilde{\boldsymbol{\theta}}_{\hat{l}}+2 \boldsymbol{z}_{\hat{l}, k^{(l)}} \tilde{\boldsymbol{\theta}}_{\hat{l}}+c_{\hat{l}, k^{(l)}}}{\tilde{\boldsymbol{\theta}}_{\hat{l}}^{T} \hat{\boldsymbol{Z}}_{\hat{l}, k^{(l)}} \tilde{\boldsymbol{\theta}}_{\hat{l}}+2 \hat{\boldsymbol{z}}_{\hat{l}, k^{(l)}} \tilde{\boldsymbol{\theta}}_{\hat{l}}+\hat{c}_{\hat{l}, k^{(l)}}} .
$$

In (17), both $\boldsymbol{Z}_{\hat{l}, k^{(l)}}$ and $\boldsymbol{Z}_{\hat{l}, k^{(l)}}$ belong to $\mathbb{R}^{2 M_{\hat{\imath}} \times 2 M_{\hat{\imath}}}$ and are positive semi-definite, $c_{\hat{l}, k^{(l)}}>0$ and $\hat{c}_{\hat{l}, k^{(l)}}>0$. To convert the problem (P3.1) in (6) to a DCCP optimization problem, we further define

$$
\begin{aligned}
u_{k^{(l)}} & \triangleq \tilde{\boldsymbol{\theta}}_{\hat{l}}^{T} \boldsymbol{Z}_{\hat{l}, k^{(l)}} \tilde{\boldsymbol{\theta}}_{\hat{l}}+2 \boldsymbol{z}_{\hat{l}, k^{(l)}} \tilde{\boldsymbol{\theta}}_{\hat{l}}+c_{\hat{l}, k^{(l)}}, \\
v_{k^{(l)}} & \triangleq \tilde{\boldsymbol{\theta}}_{\hat{l}}^{T} \hat{\boldsymbol{Z}}_{\hat{l}, k^{(l)}} \tilde{\boldsymbol{\theta}}_{\hat{l}}+2 \hat{\boldsymbol{z}}_{\hat{l}, k^{(l)}} \tilde{\boldsymbol{\theta}}_{\hat{l}}+\hat{c}_{\hat{l}, k^{(l)}}, \quad \forall l, k, \\
s & \triangleq \sum_{l=1}^{L} \sum_{k=1}^{M_{l}} \alpha_{k^{(l)}} \log _{2}\left(v_{k^{(l)}}\right) .
\end{aligned}
$$

The problem (P3.1) in (14) is then equivalent to the following optimization problem.

$$
\begin{aligned}
& \text { (P3.2): } \max _{\tilde{\boldsymbol{\theta}}_{\hat{l}}, \boldsymbol{u}, \boldsymbol{v}, s} \sum_{l=1}^{L} \sum_{k=1}^{M_{l}} \alpha_{k^{(l)}} \log _{2}\left(u_{k^{(l)}}+v_{k^{(l)}}\right)-s \\
& \text { s.t. } \quad u_{k^{(l)}}=\tilde{\boldsymbol{\theta}}_{\hat{l}}^{T} \boldsymbol{Z}_{\hat{l}, k^{(l)}} \tilde{\boldsymbol{\theta}}_{\hat{l}}+2 \boldsymbol{z}_{\hat{l}, k^{(l)}} \tilde{\boldsymbol{\theta}}_{\hat{l}}+c_{\hat{l}, k^{(l)}} \\
& v_{k^{(l)}}=\tilde{\boldsymbol{\theta}}_{\hat{l}}^{T} \hat{\boldsymbol{Z}}_{\hat{l}, k^{(l)}} \tilde{\boldsymbol{\theta}}_{\hat{l}}+2 \hat{\boldsymbol{z}}_{\hat{l}, k^{(l)}} \tilde{\boldsymbol{\theta}}_{\hat{l}}+\hat{c}_{\hat{l}, k^{(l)}} \\
& u_{k^{(l)}} \geq v_{k^{(l)}}\left(2^{R_{k^{(l)}} / B}-1\right), \quad \forall k, l, \\
& s=\sum_{l=1}^{L} \sum_{k=1}^{M_{l}} \alpha_{k^{(l)}} \log _{2}\left(v_{k^{(l)}}\right), \\
&\left(\boldsymbol{\theta}_{\hat{l}}^{(r)}\right)^{2}+\left(\boldsymbol{\theta}_{\hat{l}}^{(i)}\right)^{2}=1,
\end{aligned}
$$

where $\boldsymbol{u}, \boldsymbol{v} \in \mathbb{R}^{K \times 1}$ have their elements as $\left\{u_{k^{(l)}}\right\},\left\{v_{k^{(l)}}\right\}$, respectively; $s \in \mathbb{R}$ is a slack variable; $\boldsymbol{\theta}_{\hat{l}}^{(r)}$ and $\boldsymbol{\theta}_{\hat{l}}^{(i)}$ are defined in (16). Note that the objective function and constraints of the problem (19) satisfy the requirements of a DCCP problem [27]. If it is feasible, the local optimality is guaranteed by CVXPY/DCCP solvers [26], [27]. Since the problem (P3.2) in (19) and (P3.1) in (14) are equivalent, after solving (P3.3), we can obtain the locally optimal solution for (14) as $\boldsymbol{\theta}_{\hat{l}}^{*}=\tilde{\boldsymbol{\theta}}_{\hat{l}}\left[1: M_{t} / 2\right]+j \tilde{\boldsymbol{\theta}}_{\hat{l}}\left[M_{t} / 2+1: M_{t}\right]$ where $j \triangleq \sqrt{-1}$.

\section{Proposed Iterative AO Algorithm}

The proposed iterative AO algorithm to solve the optimization problem (P1) in (6) is summarized in Algorithm 1, where we define $\tilde{\boldsymbol{\Theta}}^{(t)}=\left[\tilde{\boldsymbol{\theta}}_{1}^{(t)}, \tilde{\boldsymbol{\theta}}_{2}^{(t)}, \ldots, \tilde{\boldsymbol{\theta}}_{L}^{(t)}\right]$ as the phase-shift matrix at the $t$-th iteration. The convergence to the locally optimal solution of the problem (6) is formally stated in the following theorem.

Theorem 2. Algorithm 1 is guaranteed to converge to a locally optimal solution of the problem (P1) in (6).

Proof: See Appendix B.
Algorithm 1 Iterative algorithm to solve the non-convex optimization problem (P1) in (6).

1: Input: The previous output $\left\{\boldsymbol{w}_{k^{(l)}}^{(t-1)}, \tilde{\boldsymbol{\theta}}_{l}^{(t-1)}\right\}$.

2: Initialize $t=1,\left\{\boldsymbol{w}_{k^{(l)}}^{(0)}, \tilde{\boldsymbol{\theta}}_{l}^{(0)}\right\},\left\{\alpha_{k^{(l)}}\right\}$, maximum number of iteration, and tolerance $\xi, \epsilon_{2}>0$.

3: Compute the weighted sum-rate $\Gamma^{(0)}\left(\boldsymbol{W}^{(0)}, \tilde{\boldsymbol{\Theta}}^{(0)}\right)=$ $\sum_{l=1}^{L} \sum_{k=1}^{M_{l}} \alpha_{k^{(l)}} \log _{2}\left(1+\gamma_{k^{(l)}}^{(0)}\left(\boldsymbol{w}_{k^{(l)}}^{(0)}, \tilde{\boldsymbol{\Theta}}^{(0)}\right)\right)$.

4: Repeat

5: $\quad$ Obtain $\boldsymbol{w}_{k(l)}^{(t)}$ from $\left\{\tilde{\boldsymbol{\theta}}_{l}^{(t-1)}\right\}$ by solving (8);

6: $\quad$ For each $l \in \mathcal{L}:$

7: Obtain $\tilde{\boldsymbol{\theta}}_{\hat{l}}^{(t)}$ by iteratively solving (19) using the CVXPY/DCCP solver [26], [27] with $\boldsymbol{w}_{k^{(l)}}=\boldsymbol{w}_{k^{(l)}}^{(t)}$, $\tilde{\boldsymbol{\theta}}_{l}=\tilde{\boldsymbol{\theta}}_{l}^{(t)}$ for $l<\hat{l}$, and $\tilde{\boldsymbol{\theta}}_{l}=\tilde{\boldsymbol{\theta}}_{l}^{(t-1)}$ for $l>\hat{l}$ and tolerance $\epsilon_{2}$;

8: Compute the weighted sum-rate $\Gamma^{(t)}\left(\boldsymbol{W}^{(t)}, \tilde{\boldsymbol{\Theta}}^{(t)}\right)=$ $\sum_{l=1}^{L} \sum_{k=1}^{M_{l}} \alpha_{k^{(l)}} \log _{2}\left(1+\gamma_{k^{(l)}}^{(t)}\left(\boldsymbol{w}_{k^{(l)}}^{(t)}, \tilde{\boldsymbol{\Theta}}^{(t)}\right)\right)$;

9: $\quad$ If

10: $\quad\left|\Gamma^{(t)}-\Gamma^{(t-1)}\right|<\xi$;

11: Then

12: $\quad$ Set $\left\{\boldsymbol{w}_{k^{(l)}}^{*}, \tilde{\boldsymbol{\theta}}_{l}^{*}\right\}=\left\{\boldsymbol{w}_{k^{(l)}}^{(t)}, \tilde{\boldsymbol{\theta}}_{l}^{(t)}\right\}$ and terminate.

13: Otherwise

14: $\quad$ Update $t \leftarrow t+1$ and continue.

15: Output: The locally optimal solution $\hat{\chi}^{*}=\left\{\boldsymbol{w}_{k^{(l)}}^{*}, \tilde{\boldsymbol{\theta}}_{l}^{*}\right\}$.

\section{E. Complexity Analysis}

The complexity of solving the optimization problem in (6) is the combined complexity of solving the beamforming weights as in Section III-A and $L$ optimization problems in (9). As shown in the previous subsection, the complexity of obtaining the weights in (8) is $\mathcal{O}\left(N^{3}\right)$. Similar to the analysis in [13], the complexity of solving the $l$-th optimization problem in (19), which has $2 K+2 M_{l}$ variables and $3 K+M_{l}$ constraints, is $\mathcal{O}\left(\left(2 K+2 M_{l}\right)^{2.5}\left(3 K+M_{l}\right) \log _{2}\left(1 / \epsilon_{2}\right)\right) \approx \mathcal{O}\left(\left(K^{3.5}+\right.\right.$ $\left.\left.M_{l}^{3.5}\right) \log _{2}\left(1 / \epsilon_{2}\right)\right)$, where $\epsilon_{2}$ is the accuracy of the DCCP method for solving (19). Since the AO algorithm iteratively solves each of these $L$ problems, the corresponding complexity is $\mathcal{O}\left(\left(L K^{3.5}+\sum_{l=1}^{L} M_{l}^{3.5}\right) \log _{2}\left(1 / \epsilon_{2}\right)\right)$. Therefore, the total complexity of Algorithm 1 is $\mathcal{O}\left(I_{1}\left(N^{3}+\left(L K^{3.5}+\right.\right.\right.$ $\left.\left.\sum_{l=1}^{L} M_{l}^{3.5}\right) \log _{2}\left(1 / \epsilon_{2}\right)\right)$, where $I_{1}$ is the required number of iterations for the outer alternative algorithm to converge. When all RSs have the same number of elements $M_{l}=M_{1}$ for all $l$, the complexity is given by $\mathcal{O}\left(I_{1}\left(N^{3}+L\left(K^{3.5}+\right.\right.\right.$ $\left.M_{1}^{3.5}\right) \log _{2}\left(1 / \epsilon_{2}\right)$ ). Our simulations in Section V show that Algorithm 1 always converges after about $I_{1} \sim 100$ iterations under our considered setup.

\section{Minimum-Rate Maximization}

In this section, we aim to maximize the minimum achievable rate among all users by optimizing the phase-shifts of all RSs as well as the beamforming vectors at the BS for all groups of users [18]. The problem is formally written as follows

(P4): $\max _{\left\{\boldsymbol{w}_{k^{(l)}}\right\},\left\{\boldsymbol{\theta}_{l}\right\}} \min _{\{l, k\}} \log _{2}\left(1+\gamma_{k^{(l)}}\right)$ 
where $\gamma_{k^{(l)}}$ is given in (5).

Note that the authors of [16] also aimed to maximize the minimum achievable rate among all users but for a different setting. Specifically, as aforementioned, only two RSs and one group of users were considered in [16]. For that, this work did not consider the interference as well as the secondary reflections amongst more than two RSs as in our current work.

It can be easily observed that the constraints $\left|\theta_{l, j}\right|=1$ are non-convex. For that the feasible region of the problem ( $\mathrm{P} 4)$ is non-convex, so is the optimization problem [18].

As (P4) is a non-convex problem, a common approach in the literature to solve it is to design an $\mathrm{AO}$ algorithm to find a suboptimal solution. First, we fix all the RSs phase-shifts $\left\{\boldsymbol{\theta}_{l}\right\}_{l=1}^{L}$ to optimize the receive beamforming $\left\{\boldsymbol{w}_{k^{(l)}}\right\}$ for all users $k^{(l)}$. In this case, the effective channel of each user $\boldsymbol{h}_{k^{(l)}}$ in (2) is fixed and thus problem (P4) is reduced to $K$ subproblems. Each of the sub-problem is to solve for the receive beamforming weights to maximize the SINR of the $k^{(l)}$-th user, and the optimal MMSE-based solution is given in (8) as in Section III-A.

Then, for each $\hat{l} \in \mathcal{L}$, the corresponding $\boldsymbol{\theta}_{\hat{l}}$ is optimized while the $\left\{\boldsymbol{w}_{k^{(l)}}\right\}$ 's and all other $\left\{\boldsymbol{\theta}_{\hat{l}}\right\}_{\hat{l} \neq \hat{l}}^{L}$ are fixed. Although the AO method is also used in [16] and other works, in our case, solving (P4) is not straightforward due to the new intricacy introduced by multiple RSs, secondary reflections amongst RSs, and multiple groups of users. In the next steps, we focus on optimizing the phase-shifts given the optimal MMSE beamforming vectors.

\section{A. Optimize $\left\{\boldsymbol{\theta}_{l}\right\}_{l=1}^{L}$ for Fixed $\left\{\boldsymbol{w}_{k^{(l)}}\right\}$ 's}

We note that maximizing the minimum $\log _{2}\left(1+\gamma_{k^{(l)}}\right)$ over $\{l, k\}$ is equivalent to maximizing the minimum SINR $\gamma_{k^{(l)}}$ over $\{l, k\}$. Hence, for fixed $\left\{\boldsymbol{w}_{k^{(l)}}\right\}$ 's, the problem (P4) in (20) is equivalent to the following optimization problem

$$
\begin{aligned}
& \text { (P4.1): } \quad \max _{\left\{\boldsymbol{\theta}_{l}\right\}, \delta} \delta \\
& \text { s.t. } \gamma_{k^{(l)}} \geq \delta, \text { for all } l \in \mathcal{L}, \quad k \in \mathcal{K}_{l}, \\
& \quad\left|\theta_{l, j}\right|=1, \text { for all } l \in \mathcal{L}, \quad j \in \mathcal{M}_{l},
\end{aligned}
$$

where $\delta$ is a slack variable and $\gamma_{k^{(l)}}$ is given in (5).

The problem (P4.1) is non-convex because its constraints $\left|\theta_{l, j}\right|=1$ make its feasible region a non-convex set. We proceed to apply the AO algorithm to solve for (P4.1) by alternately optimizing for each $\boldsymbol{\theta}_{l}$ while considering all the other phase-shifts to be fixed as detailed in the following subsection.

\section{B. Optimize $\boldsymbol{\theta}_{\hat{l}}$ for Fixed $\left\{\boldsymbol{\theta}_{l^{\prime}}\right\}_{l^{\prime} \neq \hat{l}}$ and Fixed $\left\{\boldsymbol{w}_{k^{(l)}}\right\}$ 's}

For fixed $\left\{\boldsymbol{\theta}_{l^{\prime}}\right\}_{\hat{l}=1, l^{\prime} \neq \hat{\imath}}^{L}$ and $\left\{\boldsymbol{w}_{k^{(l)}}\right\}$ 's, the problem (P3) is reduced to the following problem

$$
\begin{array}{ll}
\text { (P4.2) : } & \max _{\boldsymbol{\theta}_{\hat{l}}, \delta} \\
\text { s.t. } & \gamma_{k^{(l)}} \geq \delta \text { for all } l \in \mathcal{L}, k \in \mathcal{K}_{l}, \\
& \left|\theta_{\hat{l}, j}\right|=1, \text { for all } j \in \mathcal{M}_{\hat{l}},
\end{array}
$$

where $\hat{l} \in \mathcal{L}$ is a predefined value. Note that (P4.2) is also a non-convex problem due to the unity constraints on $\left|\theta_{\hat{l}, j}\right|$.
Using Theorem 1, when only $\boldsymbol{\theta}_{\hat{l}}$ is a design variable, the problem (P4.2) in (22) can be rewritten as follows

$$
\begin{aligned}
\max _{\boldsymbol{\theta}_{\hat{l}}, \delta} & \delta \\
\text { s.t. } & \left|\boldsymbol{q}_{\hat{l}, k^{(l)}}^{H} \boldsymbol{\theta}_{\hat{l}}+p_{\hat{l}, k^{(l)}}\right|^{2} \\
& \geq \delta \sum_{\substack{(\hat{l}, \tilde{k}) \\
\forall(l, k)}}\left|\boldsymbol{q}_{\hat{l}, \tilde{k}^{(\tilde{l})}}^{H} \boldsymbol{\theta}_{\hat{l}}+p_{\hat{l}, \tilde{k}^{(\tilde{l})}}\right|^{2}+\delta \sigma_{k^{(l)}}^{2} \\
& \left|\theta_{\hat{l}, j}\right|=1, \forall j \in \mathcal{M}_{\hat{l}}, \text { and for all }(l, k) .
\end{aligned}
$$

We can then rewrite

$$
\begin{aligned}
\left|\boldsymbol{q}_{\hat{l}, k^{(l)}}^{H} \boldsymbol{\theta}_{\hat{l}}+p_{\hat{l}, k^{(l)}}\right|^{2} & =\tilde{\boldsymbol{\theta}}_{\hat{l}}^{H} \boldsymbol{B}_{\hat{l}, k^{(l)}} \tilde{\boldsymbol{\theta}}_{\hat{l}}+\left|p_{\hat{l}, k^{(l)}}\right|^{2} \\
& =\operatorname{Tr}\left(\boldsymbol{B}_{\hat{l}, k^{(l)}} \tilde{\boldsymbol{\theta}}_{\hat{l}} \tilde{\boldsymbol{\theta}}_{\hat{l}}^{H}\right)+\left|p_{\hat{l}, k^{(l)}}\right|^{2},
\end{aligned}
$$

where

$$
\boldsymbol{B}_{\hat{l}, k^{(l)}} \triangleq\left[\begin{array}{cc}
\boldsymbol{q}_{\hat{l}, k^{(l)}} \boldsymbol{q}_{\hat{l}_{, k}^{(l)}}^{H} & p_{\hat{l}, k^{(l)}} \boldsymbol{q}_{\hat{l}, k^{(l)}} \\
p_{\hat{l}, k^{(l)}}^{H} \boldsymbol{q}_{\hat{l}, k^{(l)}}^{H} & 0
\end{array}\right], \quad \tilde{\boldsymbol{\theta}}_{\hat{l}} \triangleq\left[\begin{array}{c}
\boldsymbol{\theta}_{\hat{l}} \\
s
\end{array}\right],
$$

with $s$ is an auxiliary variable ( $s=1$ to be exact but we relax this condition and assume $|s|=1$ ). Let's define $\Psi_{l} \triangleq \tilde{\boldsymbol{\theta}}_{l} \tilde{\boldsymbol{\theta}}_{l}^{H}$. We then have $\Psi_{l} \succeq 0$ and $\operatorname{rank}\left(\Psi_{l}\right)=1$. Since the rankone constraint, $\operatorname{rank}\left(\Psi_{l}\right)=1$, is non-convex, we relax this constraint. For that, the problem (23) is rewritten as

$$
\begin{aligned}
& \text { (P4.3) : } \max _{\Psi_{\hat{\imath}}, \delta} \delta \\
& \text { s.t. } \operatorname{Tr}\left(\boldsymbol{B}_{\hat{l}, k^{(l)}} \Psi_{\hat{l}}\right)+\left|p_{\hat{l}, k^{(l)}}\right|^{2} \geq \\
& \delta \sum_{(\tilde{l}, \tilde{k}) \neq\left(k^{(l)}\right)} \operatorname{Tr}\left(\boldsymbol{B}_{\hat{l}, \tilde{k}^{(\tilde{l})}} \Psi_{\hat{l}}\right)+ \\
& \delta\left(\sum_{(\tilde{l}, \tilde{k}) \neq\left(k^{(l)}\right)}\left|p_{\hat{l}, \tilde{k}^{(\tilde{l})}}\right|^{2}+\sigma_{k^{(l)}}^{2}\right) \\
& \Psi_{\hat{l}} \succeq 0, \quad\left[\Psi_{\hat{l}}\right]_{j j}=1, \\
& \forall j \in \mathcal{M}_{\hat{l}}+1 \text {, and for all }\left(k^{(l)}\right) \text {. }
\end{aligned}
$$

For a fixed $\delta,(\mathrm{P} 4.3)$ is a convex semidefinite program (SDP) problem and is reduced to a feasibility-check problem [16], [24]. This problem can be optimally solved by standard convex optimization solvers [25]. Therefore, (P4.3) can be efficiently solved by the bisection method; that is, we do a binary search on $\delta$ that has a feasible solution $\Psi_{\hat{l}}$. Once we obtain a globally maximum $\delta_{\text {opt }}$ (up to a certain numerical accuracy) with a $\Psi_{\hat{l}}$ solution, we can use the Gaussian randomization search to obtain a solution for $\boldsymbol{\theta}_{\hat{l}}$ [5]. Our simulation shows that the numerical solution $\Psi_{\hat{l}}$ is typically rank deficient (i.e., its maximum eigen-value is much larger than the rest), and we can almost always obtain a numerical solution $\boldsymbol{\theta}_{\hat{l}}$ such that the max-min achievable rate is within a pre-defined error, e.g., $\epsilon=0.1 \%$, relative to the globally optimal $\delta_{\text {opt }}$ of the problem (P4.3).

Because we obtain $\boldsymbol{\theta}_{\hat{\imath}}$ from $\Psi_{\hat{l}}$ using the Gaussian randomization method, there is a possibility that the solution at the $(t-1)$-th iteration is better than that at the $t$-th iteration. To guarantee that Algorithm 2 converges, we add a heuristic check as shown in lines $9-14$. That is, when the solution $\boldsymbol{\theta}_{\hat{l}}^{(t)}$ results 
in a strictly smaller value of the original objective function in the problem (P4), it will not be updated.

\section{Iterative AO Algorithm for Max-Min Achievable Rate}

The proposed iterative algorithm is summarized in Algorithm 2, in which $\boldsymbol{\Theta}^{(t)}=\left[\boldsymbol{\theta}_{1}^{(t)}, \boldsymbol{\theta}_{2}^{(t)}, \ldots, \boldsymbol{\theta}_{L}^{(t)}\right]$ is the phase-shift matrix at the iteration $t$. The convergence of the algorithm is provided in the following theorem [18].

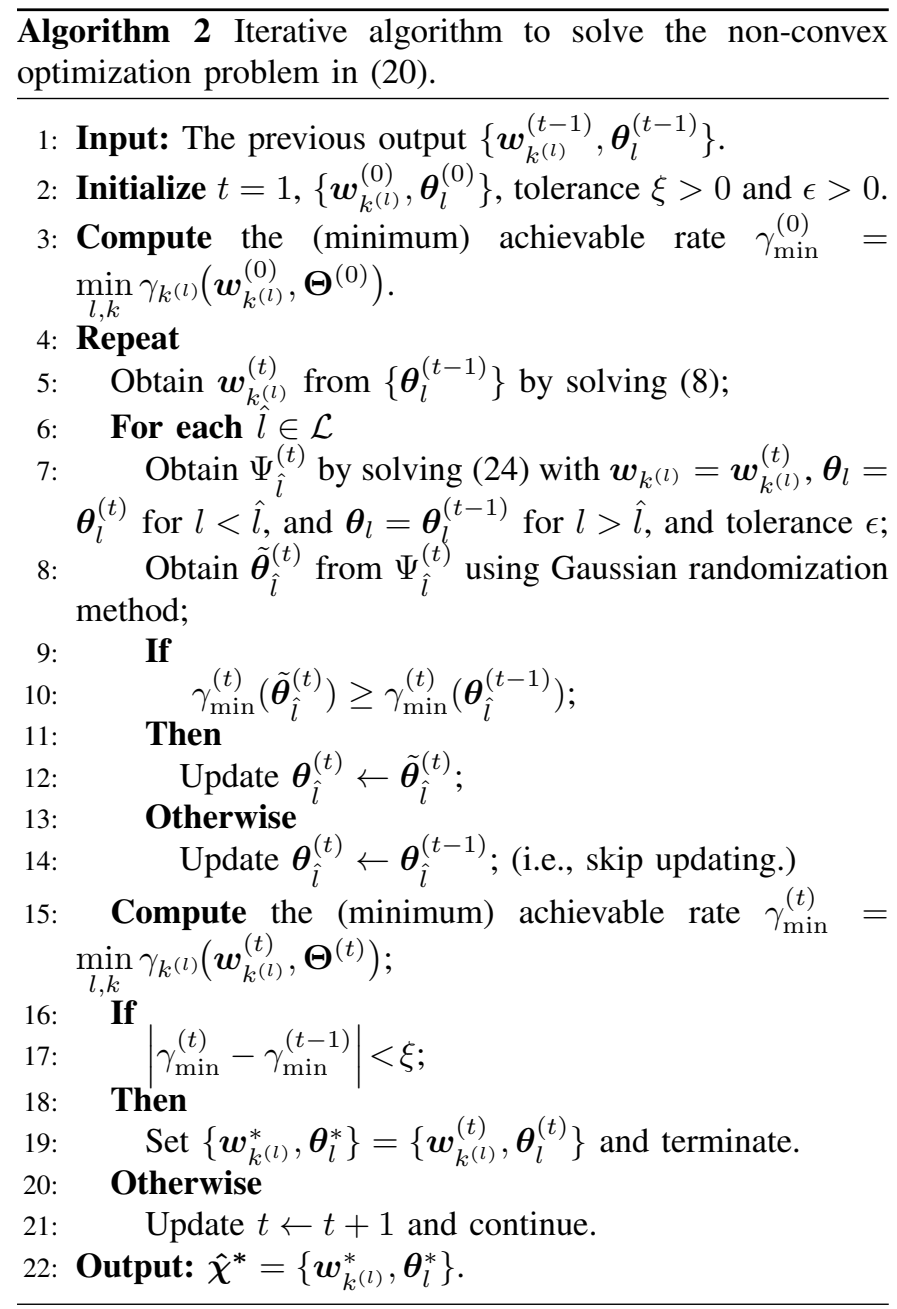

Theorem 3. The objective value $\gamma_{\min }^{(t)}$ as shown in Algorithm 2 is monotonically non-decreasing (and always bounded above) and thus the algorithm is guaranteed to converge.

Proof: See Appendix C.

\section{Complexity Analysis}

The complexity of solving optimization problem (20) is the combined complexity of solving the beamforming weights as shown in (8) whose complexity is mainly due to the computation of the inverse of an $N \times N$ matrix, hence $\mathcal{O}\left(N^{3}\right)$. Similar to the analysis in [13], the complexity of solving the $l$-th SDP optimization problem in (24) with the bisection method, which has $M_{l}^{2}$ variables, is $\mathcal{O}\left(M_{l}^{4.5} \log (1 / \epsilon)\right)$ where $\epsilon$, as shown in Algorithm 2, is the accuracy of the bisection search. Note that the algorithm alternately solves $L$ of these problems, hence the total complexity is $\mathcal{O}\left(\left(M_{1}^{4.5}+\right.\right.$ $\left.\left.\ldots+M_{L}^{4.5}\right) \log (1 / \epsilon)\right)$. This becomes $\mathcal{O}\left(L \cdot M_{1}^{4.5} \log (1 / \epsilon)\right)$ when $M_{l}=M_{1}$ for all $l$. Hence, the total complexity of Algorithm 2 is $\mathcal{O}\left(I_{2}\left(\left(M_{1}^{4.5}+\ldots+M_{L}^{4.5}\right) \log (1 / \epsilon)+N^{3}\right)\right.$, or $\mathcal{O}\left(I_{2}\left(L M_{1}^{4.5} \log (1 / \epsilon)+N^{3}\right)\right.$ when $M_{l}=M_{1}$ for all $l$, where $I_{2}$ is the required number of iterations for the outer alternative algorithm to be converged. Our simulations below show that Algorithm 2 always converges after about $I_{2} \sim 100$ iterations under our considered setup.

\section{NUMERICAL RESULTS}

In this section, we consider the uplink transmission to a BS with 16 or more antennas, using orthogonal frequency-division multiplexing (OFDM) and varying the number of RSs. The number of elements per RS is varied from 32 to 128 . The number of users aided by each RS is from 2 to 12 users. We assume that each user is allocated with $B=180 \mathrm{KHz}$ bandwidth. Thus, the noise power at the BS is assumed to be $\sigma^{2}=-174+10 \log _{10}(B) \mathrm{dBm}$. Similar to the simulation setup and assumption in [10], [20], [39], we consider a smallcell situation as shown in Fig. 2.

In this setup, we assume the RSs' locations are within $180^{\circ}$ half-space reflection of each other. Particularly, the RSs are located with equal spacing in a half circle with diameter of 20 meters (unless otherwise stated) and facing the center of the circle (see Fig. 2). The distance between the BS and the center of the circle is 50 meters. The users' locations are assumed to be uniformly distributed in a half circle with diameter $d_{r s 2 r s}$ and within the $180^{\circ}$ half-space reflection of each RS. We assume the heights of BS, RSs, and users are 15, 20 and 1.5 meters, respectively. The transmit power at each user is assumed to be the same. In the following, when the number of the RSs varies from four or six, we specify the RSs' locations as $\left(x_{r s}, y_{r s}, 1.5\right)$ in meter.

We assume a non-LOS-dominant channel model between the users and the RSs with the Rician factor $\kappa=-10 \mathrm{~dB}$. The LOS-dominant channel model between the RSs and the BS and between any two RSs has Rician factor $\kappa=5 \mathrm{~dB}$. Specifically, the channel model between a source, $s$, and a destination, $d$, is assumed to be as follows:

$$
\boldsymbol{H}_{s, d}=\sqrt{\frac{\kappa}{\kappa+1}} \overline{\boldsymbol{H}}_{s, d}+\sqrt{\frac{1}{\kappa+1}} \tilde{\boldsymbol{H}}_{s, d},
$$

where $(s, d)$ can be (User, RS), (RS, RS) or (RS, BS), $\overline{\boldsymbol{H}}_{s, d}$ denotes the LOS component and $\tilde{\boldsymbol{H}}_{s, d}$ denotes the non-LOS component. We assume a rich scattering environment and thus the elements of the non-LOS components $\tilde{\boldsymbol{H}}_{s, d}$ is modeled as complex Gaussian independent and identically distributed random variables with zero mean and unit variance, i.e., $\mathcal{C N}(0,1)$. The LOS component is modeled as a rank-one matrix as follows [10], [49]:

$$
\overline{\boldsymbol{H}}_{s, d}=\boldsymbol{a} \boldsymbol{b}^{H}
$$

where $\boldsymbol{a}_{m}=\exp \left(j \frac{2 \pi}{\lambda} d_{s}(m-1) \sin \phi_{s, l o s} \sin \theta_{d, \text { los }}\right)$ and $\boldsymbol{b}_{n}=\exp \left(j \frac{2 \pi}{\lambda} d_{d}(m-1) \sin \phi_{d, \text { los }} \sin \theta_{d, \text { los }}\right)$, where $\lambda$ is the carrier wavelength, $d_{s}, d_{d}$ are inter-antenna or inter-RSelement separation at $s$ and $d$ (which can be either the BS or 

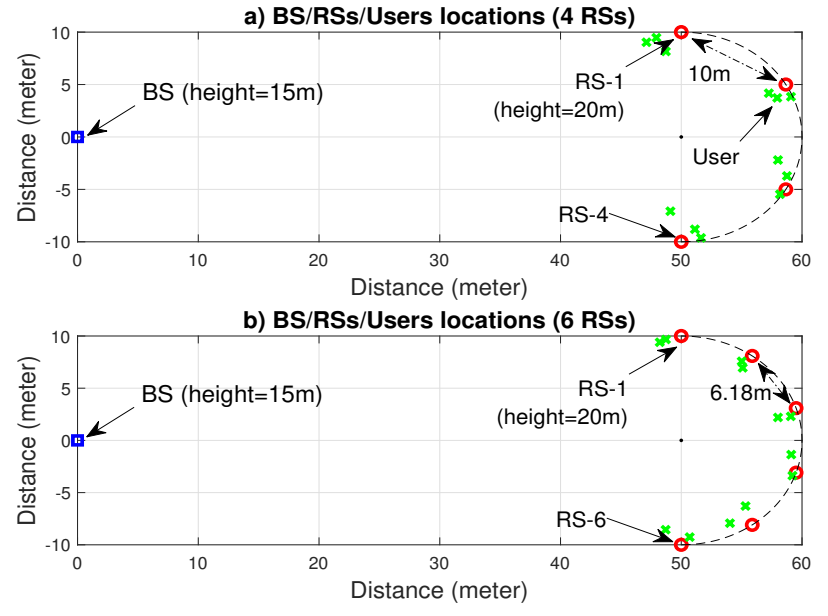

Fig. 2: Snapshots of BS, RSs and users locations with a) four RSs serving three users each or b) six RSs serving two users each.

RS), $\phi_{s, l o s}, \theta_{s, l o s}$ are LOS azimuth and elevation AoDs at $s$ and $\phi_{d, l o s}, \theta_{d, l o s}$ are LOS azimuth and elevation AoDs at $d$, respectively. In our simulation we set $d_{s}=d_{d}=0.5 \lambda$.

Consider a three-terminal reflection link, $s \rightarrow r \rightarrow d$, where $(s, r, d)$ can be (User, RS, BS), (User, RS, RS), or (RS, RS, BS), similar to [10], [19], the combined pathloss is assumed to follow the far-field product-distance model as follows:

$$
\beta_{s r d}=\beta_{s r} \cdot \beta_{r d}=\frac{C_{1}}{d_{s r}^{\alpha_{1}}} \cdot \frac{C_{2}}{d_{r d}^{\alpha_{2}}},
$$

where $C_{1}, C_{2}$ are the pathloss at one meter for the $s r$ and $r d$ links, $d_{s r}, d_{r d}$ are the distance between $s, r$ and $r, d$, and $\alpha_{1}, \alpha_{2}$ are the pathloss exponents for the $s, r$ and $r, d$ links, respectively. We assume the non-LOS-dominant channel model between users and RS and thus set $\alpha_{1}=3.0$, $C_{1}=10^{\frac{G_{s}+G_{r}-30}{10}}$, and the LOS-dominant channel model for RS-RS or RS-BS links and thus set $\alpha_{2}=2.2, C_{2}=$ $10 \frac{G_{r}+G_{d}-30}{10}$, where $G_{s}, G_{r}, G_{d}$ are the antenna/RS element gain at their respective terminal. We assume $5 \mathrm{dBi}$ gain at the BS antenna and $0 \mathrm{dBi}$ at the user antenna or the RS elements. As for the secondary reflections, the signal travels from User $\rightarrow R S_{l} \rightarrow R S_{l^{\prime}} \rightarrow B S$, and the total pathloss is modeled as the product of the far-field pathloss of each pointto-point link, that is, $\beta_{s r r^{\prime} d}=\beta_{s r} \cdot \beta_{r r^{\prime}} \cdot \beta_{r^{\prime} d}$, where $s, r, r^{\prime}, d$ denote $U$ ser, $R S_{l}, R S_{l^{\prime}}, B S$, respectively. The transmit power at each user is assumed to be the same and ranges from 10 up to $40 \mathrm{dBm}$ (i.e., $10 \mathrm{~W}$ ). Our performance result is averaged over 50 independent channel realizations.

\section{A. Sum-Rate Maximization}

First, we present the simulation results for the weighted sum-rate maximization problem. In this simulation, we assume all the users' weights are the same, i.e. $1 / K$, or equivalently we maximize the sum-rate only. The minimum rate requirement (on each user's achievable rate) is set to zero, unless otherwise stated. We focus on showing the performance gain by considering the additional secondary reflections of RSs in

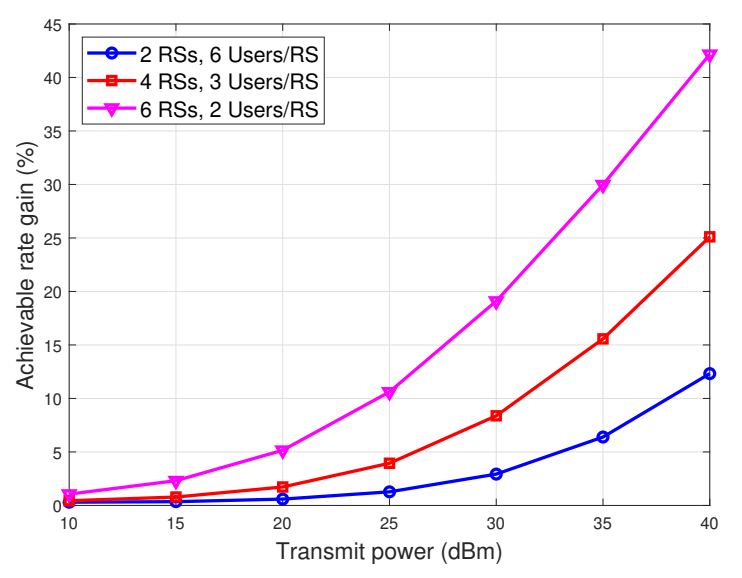

Fig. 3: Achievable sum-rate gain (percentage) versus transmit power.

our proposed design as compared to the conventional design considering the RSs' primary reflections only. Note that for fair comparison with existing designs in the literature that ignored the secondary RS reflections while optimizing the phase-shifts and beamformers, we still evaluate their performance under the same secondary reflections as for the proposed scheme.

In Fig. 3, we plot the relative gain of the achievable sumrate with and without considering the secondary reflections for a system with 2, 4, or 6 RSs (while the total number of users is the same, i.e., 12 users). The locations of the RSs in these scenarios are assumed to be equally spaced and the distance between two adjacent RSs is 15,10 and 6.18 meters for the system with 2, 4, or 6, respectively. At lower transmit power (i.e., low SNR), we observe some marginal gain, because the performance is limited by AWGN and the interference amongst RSs can be tolerated without compromising the rate. At mid to high transmit power, we can observe significant performance gain when considering the secondary reflections. For example, at a transmit power (Ptx) of $35 \mathrm{dBm}$, we see about $6.4 \%, 15.6 \%$, or $29.9 \%$ gains for the system with 2,4 , or 6 RSs, respectively. The higher gain observe with more RSs is partially due to the stronger and more secondary reflections, which is due to the smaller distance between two adjacent RSs.

In Fig. 4, we study how the number of elements per RS affects system performance with and without managing the secondary reflections. We observe that the more the number of RS elements, the higher the gain from managing and leveraging the secondary reflections. For example, at Ptx of $35 \mathrm{dBm}$, we see about $7.2 \%, 15.6 \%$, and $27.9 \%$ gains for 32, 64 and 128 elements per RS, respectively. The increase in gain is due to the fact that with more RS elements, more signal reflection energy is resulted amongst RSs, thus increasing the interference from secondary reflections.

In Fig. 5, we plot the achievable rate gain as a result of managing secondary reflections for a system with four RSs and three users while varying the distance between two adjacent RSs (i.e., $d_{r s 2 r s}$ ) and the number of RSs elements. When the distance between adjacent RSs is larger, the secondary reflection effects are smaller as expected, but with increased number 


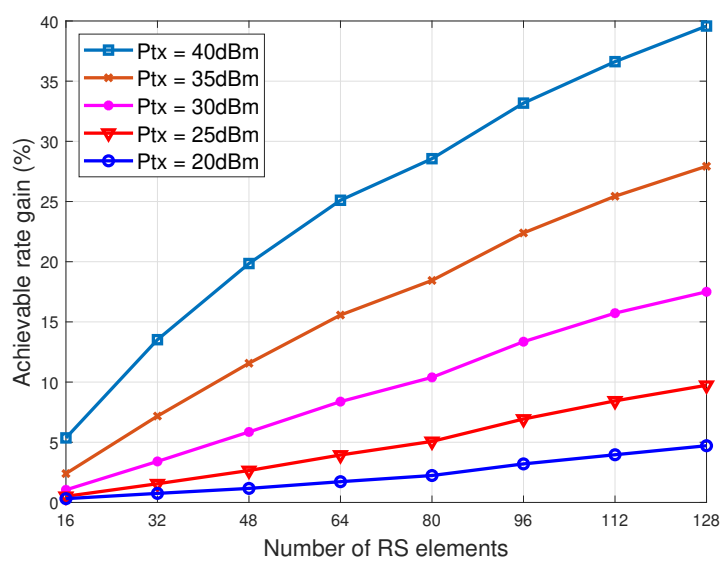

Fig. 4: Achievable sum-rate gain over the primary reflection only (percentage) versus number of RS elements.

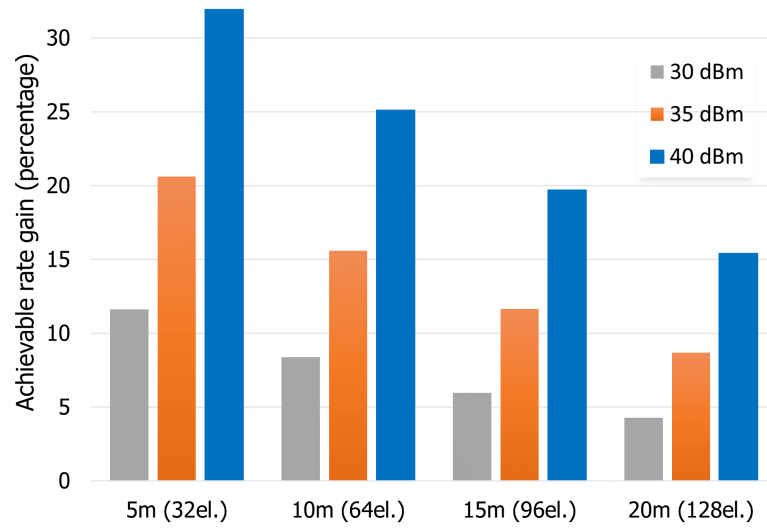

Fig. 5: Achievable sum-rate gain (percentage) versus RS distance and number of elements.

of RS elements, the gain can be significantly improved. For example, at Ptx of $35 \mathrm{dBm}$, the gains for the $(5 \mathrm{~m}, 32 \mathrm{el}$.) configuration, i.e., 5 meter (m) distance and 32 RS elements per RS and (10m, 64el.) are $20.6 \%$ and $15.6 \%$, respectively, and the gains for $(15 \mathrm{~m}, 96 \mathrm{el}$.$) and (20 \mathrm{~m}, 128 \mathrm{el}$.$) are 11.6 \%$ and $8.7 \%$, respectively.

In Fig. 6, we plot the sum-rate based on Algorithm 1 for the system with four RSs each with 3 users versus the number of iterations without early termination (i.e., we set $\xi=0$ ). We observe that the algorithm reasonably converges after about 50 iterations for $\mathrm{Ptx}=10 \mathrm{dBm}$. It requires a slightly higher number of iterations for higher Ptx. A similar trend can be observed for the max-min achievable rate based on Algorithm 2; the plot is thus omitted for brevity.

\section{B. Min-Rate Maximization}

In Fig. 7, we consider the minimum achievable rate among users under various configurations: (i) four RSs each with 3 users, (ii) two RSs each with 6 users and (iii) one RS with 12 users; that is, there are 12 users in total. The locations of the four-RS scenario are shown in Fig. 2. When there are two RSs, their locations are $\left(x_{r s}, y_{r s}\right)=(50, \pm 10)$ meters, and

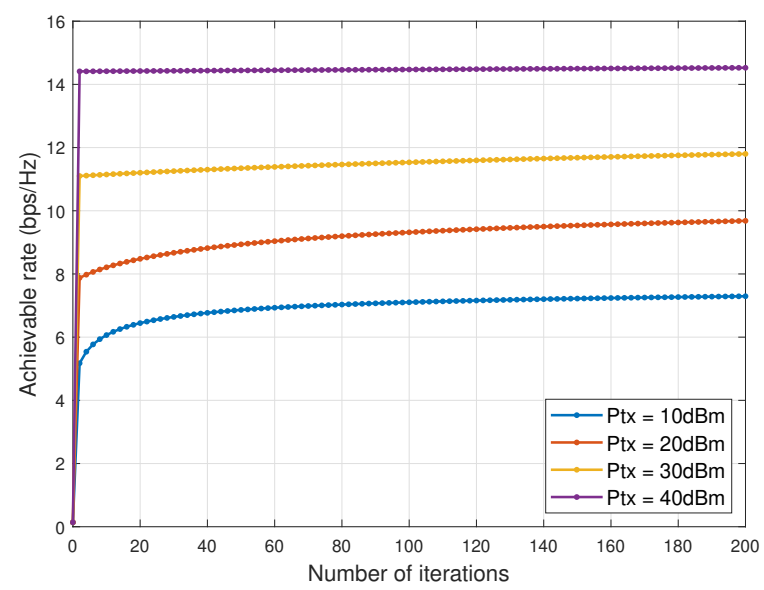

Fig. 6: Achievable rate vs number of iterations.

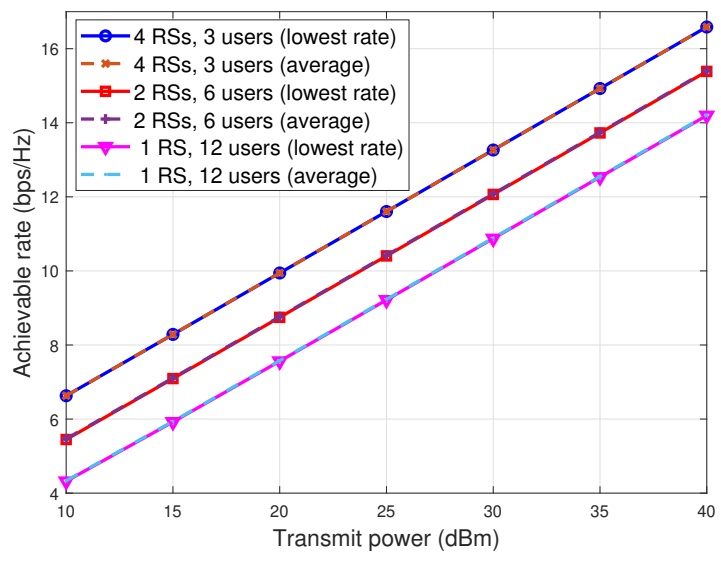

Fig. 7: The achievable min-rate among users under different RS configurations.

for the single-RS scenario, the RS is at $(50,10)$ meters. In all these configurations, each RS has 64 elements and the BS has 16 antennas. We can see that with the same number of users and receive antennas, the more RSs, the higher the minimum achievable rate. With transmit power of $30 \mathrm{dBm}$, for instance, we observe a 1.2 bits-per-second (bps)/Hz or $2.4 \mathrm{bps} / \mathrm{Hz}$ gain for a two- or four-RS scenario as compared to the single-RS system, respectively. This is because of the higher received signal power on average at the receiver antennas due to the reflections from more RSs. Note that the performance of the scenario without any RS is not shown as there is no feasible direct link between the users and the BS under our considered setup. In this figure, we also plot the average achievable rate among all users and observe that the average achievable rate has a similar trend which is not much different from the minimum achievable rate.

Fig. 8 compares the minimum achievable rate when both primary and secondary reflections are considered (as in this paper) and when only the primary reflections are considered (as in other aforementioned works [13]-[16]). We assume that the RSs are equally spaced, i.e., the distance between 


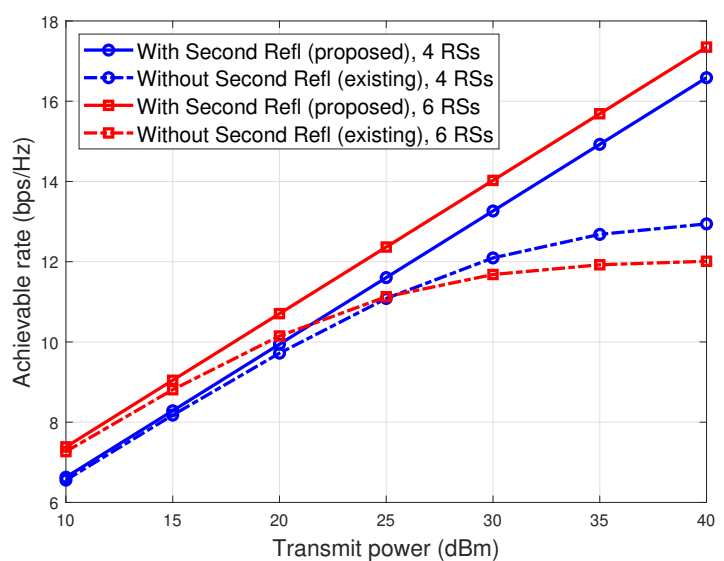

Fig. 8: The achievable min-rate among users versus transmit power.

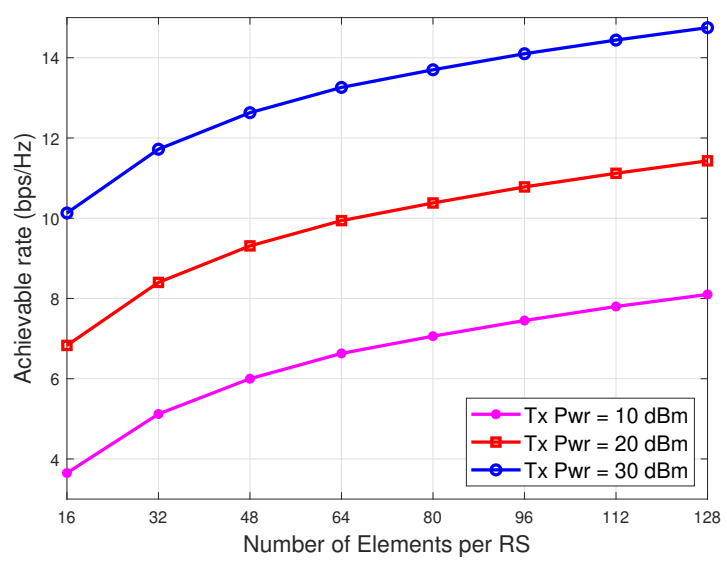

Fig. 9: The achievable min-rate among users versus number of RS elements.

two adjacent RSs for the scenarios with four and six RSs are 10 and 6.18 meters, respectively. It can be seen that, by properly managing the interference and leveraging the secondary reflections amongst RSs, the minimum achievable rate is significantly improved. For example, at transmit power of $30 \mathrm{dBm}$, we observe about 1.2 or $2.3 \mathrm{bps} / \mathrm{Hz}$ gain when exploiting the secondary reflections for a system with four or six RSs, respectively. The improvement becomes more pronounced with higher transmit power/SNR. This is because at high SNR, the secondary reflections amongst RSs become more significant while the stronger interference amongst them can be mitigated/managed under our design framework.

The management of the inter-RS interference becomes more critical for dense networks. Specifically, we observe that for a given area, without properly managing the secondary reflections, adding more RSs may actually degrade the overall system performance. This is due to the stronger interference caused by the secondary reflections from more RSs. For example, when the secondary reflections are not managed using our proposed method, we observe that the achievable rate under the six-RS scenario is even worse than that with four

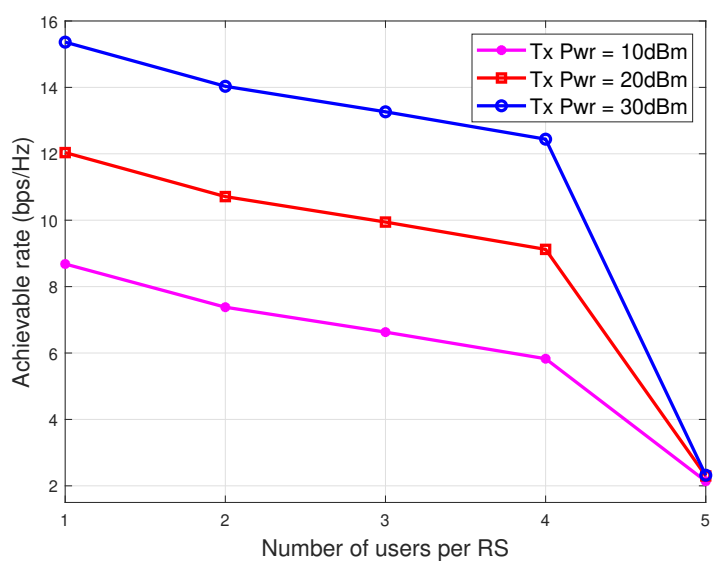

Fig. 10: The achievable min-rate among users versus number of users per RS.

RSs at the transmit power of $25.5 \mathrm{dBm}$ or higher. In contrast, by properly managing the interference using our method, the performance under the six-RS scenario always outperforms that of the four-RS scenario.

In Fig. 9, we plot the achievable rate versus different number of elements per RS for a system with four RSs, three users per RS, and a BS with 16 antennas. It is seen that the more the number of RS elements, the higher the achievable rate due to the power gain at the BS. We can see the higher the number of elements per RS, the higher the achievable rate. For example, at transmit power of $30 \mathrm{dBm}$, we can see a $1.5 \mathrm{bps} / \mathrm{Hz}$ gain for a 64-element RS system as compared to that of the 32element RS one, and a $1.4 \mathrm{bps} / \mathrm{Hz}$ gain is observed for 128 elements versus 64 elements.

In Fig. 10, we plot the achievable rate versus the number of users per RS for four RSs each with 64 elements and a BS with 16 antennas. It can be seen that when more users access the same bandwidth, the lower is the minimum achievable rate, whereas the sum-rate for all users is actually increased (before reaching its peak at 4 users per RS). However, when the total number of users is $5 \times 4=20$ (or higher) which is greater than the number of antennas at the BS, which is 16 , the achievable rate is significantly lower due to the lack of sufficient spatial channel diversity.

In Fig. 11, we plot the achievable rate versus the number of receive antennas at the BS for four RSs of 64 elements each and 3 users per RS. It shows that with more receive antennas at the BS, the higher is the minimum achievable rate. For example, at transmit power of $30 \mathrm{dBm}$, we observe about 1.7 $\mathrm{bps} / \mathrm{Hz}$ or $0.9 \mathrm{bps} / \mathrm{Hz}$ gain when there are 64 or 32 antennas as compared to 16 antennas, respectively. We can also observe that the rate for 8 antennas is significantly lower than that for 12 or 16 antennas due to the lack of sufficient spatial diversity since there are totally 12 users in this case.

\section{CONCLUSION}

In this paper, we considered an uplink multi-IRS/RIS aided multi-user MIMO communication system, where the phaseshifts of all elements at all the RSs and the received beam- 


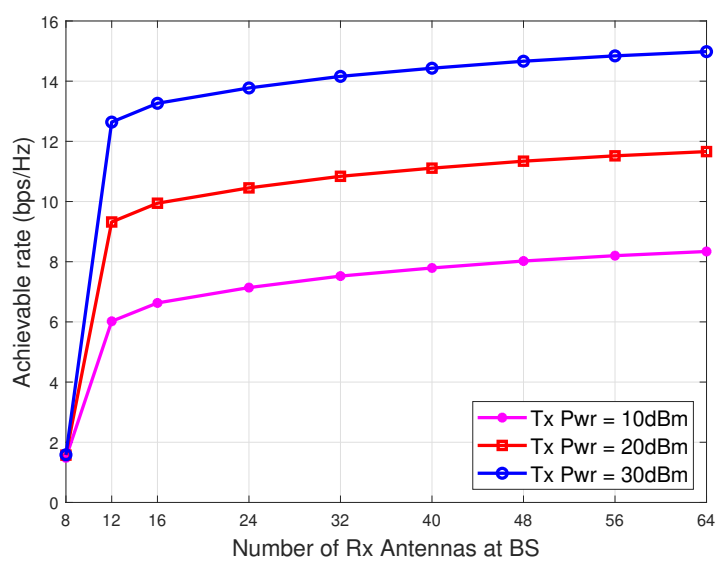

Fig. 11: The achievable min-rate among users versus number of receiver $(\mathrm{Rx})$ antennas.

forming at the BS were jointly optimized. We formulated and solved two optimization problems using AO algorithms. The numerical results show that by managing the interference and leveraging the secondary reflections amongst closely-deployed RSs, the system throughput can be significantly improved in terms of the sum-rate or the minimum rate among the users, especially when more RSs or more elements per RS are employed.

\section{APPENDIX A}

\section{PROOF OF THEOREM 1}

First, we rewrite the $\boldsymbol{h}_{k^{(l)}}$ as shown in (2) as a function of $\boldsymbol{\theta}_{\hat{l}}$. When $l \neq \hat{l}$, we have

$$
\begin{aligned}
\boldsymbol{h}_{k^{(l)}}= & \sum_{l^{\prime}=1}^{L} \boldsymbol{R}_{l^{\prime}, k^{(l)}} \boldsymbol{\theta}_{l^{\prime}}+\sum_{l^{\prime} \neq l}^{L} \sum_{j=1}^{M_{l}} \boldsymbol{Q}_{l^{\prime}, k^{(l)}, j} \boldsymbol{\theta}_{l^{\prime}} \theta_{l, j} \\
= & \boldsymbol{R}_{\hat{l}, k^{(l)}} \boldsymbol{\theta}_{\hat{l}}+\sum_{l^{\prime}=1, l^{\prime} \neq \hat{l}}^{L} \boldsymbol{R}_{l^{\prime}, k^{(l)}} \boldsymbol{\theta}_{l^{\prime}} \\
& +\left(\sum_{j=1}^{M_{l}} \boldsymbol{Q}_{\hat{l}, k^{(l)}, j^{\prime}} \theta_{l, j}\right) \boldsymbol{\theta}_{\hat{l}}+\sum_{j=1}^{M_{l}}\left(\sum_{l^{\prime} \neq l=1}^{L} \boldsymbol{Q}_{l^{\prime}, k^{(l)}, j} \boldsymbol{\theta}_{l^{\prime}}\right) \theta_{l, j} \\
= & \left(\boldsymbol{R}_{\hat{l}, k^{(l)}}+\boldsymbol{T}_{\hat{l}, k^{(l)}}\right) \boldsymbol{\theta}_{\hat{l}}+\left(\boldsymbol{U}_{\hat{l}, k^{(l)}}+\boldsymbol{S}_{\hat{l}, k^{(l)}} \boldsymbol{\theta}_{l}\right), \quad(28)
\end{aligned}
$$

where we have defined

$$
\boldsymbol{S}_{\hat{l}, k^{(l)}} \triangleq\left[\boldsymbol{s}_{\hat{l}, k^{(l)}, 1}, \ldots, \boldsymbol{s}_{\hat{l}, k^{(l)}, M_{l}}\right],
$$

with $\boldsymbol{s}_{\hat{l}, k^{(l)}, j} \triangleq \sum_{l^{\prime} \neq \hat{l}, l^{\prime} \neq l} \boldsymbol{Q}_{l^{\prime}, k^{(l)}, j} \boldsymbol{\theta}_{l^{\prime}}$,

$$
\boldsymbol{T}_{l^{\prime}, k^{(l)}} \triangleq \sum_{j=1}^{M_{l^{\prime}}} \boldsymbol{Q}_{l^{\prime}, k^{(l)}, j} \theta_{l^{\prime}, j}, \text { and } \boldsymbol{U}_{l^{\prime}, k^{(l)}} \triangleq \sum_{\substack{l^{\prime}=1 \\ l^{\prime} \neq \hat{l}}}^{L} \boldsymbol{R}_{l^{\prime}, k^{(l)}} \boldsymbol{\theta}_{l^{\prime}}
$$

When $l=\hat{l}$, we also have

$$
\begin{aligned}
\boldsymbol{h}_{k^{(l)}} & =\boldsymbol{R}_{\hat{l}, k^{(l)}} \boldsymbol{\theta}_{\hat{l}}+\sum_{\substack{l^{\prime}=1 \\
l^{\prime} \neq \hat{l}}}^{L} \boldsymbol{R}_{l^{\prime}, k^{(l)}} \boldsymbol{\theta}_{l^{\prime}}+\sum_{j=1}^{M_{\hat{l}}} \sum_{l^{\prime} \neq \hat{l}}^{L} \boldsymbol{Q}_{l^{\prime}, k(l), j} \boldsymbol{\theta}_{l^{\prime}} \theta_{\hat{l}, j} \\
& =\boldsymbol{R}_{\hat{l}, k^{(l)}} \boldsymbol{\theta}_{\hat{l}}+\boldsymbol{U}_{\hat{l}, k^{(l)}}+\boldsymbol{S}_{\hat{l}, k^{(l)}} \boldsymbol{\theta}_{\hat{l}} \\
& =\left(\boldsymbol{R}_{\hat{l}, k^{(l)}}+\boldsymbol{S}_{\hat{l}, k^{(l)}}\right) \boldsymbol{\theta}_{\hat{l}}+\boldsymbol{U}_{\hat{l}, k^{(l)}} .
\end{aligned}
$$

Now by substituting (28) and (31) into (5), we can obtain (10) with $\boldsymbol{q}_{\hat{l}, k^{(l)}}$ and $p_{\hat{l}, k^{(l)}}$ given in (12) and (13), respectively.

\section{APPENDIX B}

PROOF OF THEOREM 2

First, we prove that the sequence $\Gamma^{(t)}$ is non-decreasing, or $\Gamma^{(t)} \geq \Gamma^{(t-1)}$ for all $t>0$. In step 5 of Algorithm 1 , we obtain $\boldsymbol{w}_{k^{(l)}}^{(t)}$ from $\left\{\tilde{\boldsymbol{\theta}}_{l}^{(t-1)}\right\}$ by solving (8), which is the optimal MMSE solution. Thus, we have $\tilde{\gamma}_{k^{(l)}}^{(t)}\left(\boldsymbol{w}_{k^{(l)}}^{(t)}, \tilde{\boldsymbol{\Theta}}^{(t-1)}\right) \geq$ $\gamma_{k^{(l)}}^{(t-1)}\left(\boldsymbol{w}_{k^{(l)}}^{(t-1)}, \tilde{\boldsymbol{\Theta}}^{(t-1)}\right)$, for all $l, k$. It follows that the weighted sum-rate satisfies

$$
\Gamma^{(t)}\left(\boldsymbol{W}^{(t)}, \tilde{\boldsymbol{\Theta}}^{(t-1)}\right) \geq \Gamma^{(t-1)}\left(\boldsymbol{W}^{(t-1)}, \tilde{\boldsymbol{\Theta}}^{(t-1)}\right) .
$$

Next, in step 6 and 7 , for each $\hat{l} \in \mathcal{L}$, we obtain $\tilde{\boldsymbol{\theta}}_{\hat{l}}^{(t)}$ by solving (19) using CVXPY/DCCP solvers with $\tilde{\boldsymbol{\theta}}_{\hat{\hat{l}}}^{(t-1)}$ as the initial value (while the other variables are fixed). It is evident that

$$
\begin{gathered}
\Gamma^{(t)}\left(\boldsymbol{W}^{(t)}, \tilde{\boldsymbol{\theta}}_{1}^{(t)}, \ldots, \boldsymbol{\theta}_{\hat{l}-1}^{(t)}, \boldsymbol{\theta}_{\hat{l}}^{(t)}, \tilde{\boldsymbol{\theta}}_{\hat{l}+1}^{(t-1)}, \ldots, \tilde{\boldsymbol{\theta}}_{L}^{(t-1)}\right) \\
\geq \Gamma^{(t)}\left(\boldsymbol{W}^{(t)}, \tilde{\boldsymbol{\theta}}_{1}^{(t)}, \ldots, \boldsymbol{\theta}_{\hat{l}-1}^{(t)}, \boldsymbol{\theta}_{\hat{l}}^{(t-1)}, \tilde{\boldsymbol{\theta}}_{\hat{l}+1}^{(t-1)}, \ldots, \tilde{\boldsymbol{\theta}}_{L}^{(t-1)}\right),
\end{gathered}
$$

for all $\hat{l} \in \mathcal{L}$. Combining the above, we have

$$
\Gamma^{(t)}\left(\boldsymbol{W}^{(t)}, \tilde{\boldsymbol{\theta}}_{1}^{(t)}, \ldots, \tilde{\boldsymbol{\theta}}_{L}^{(t)}\right) \geq \Gamma^{(t)}\left(\boldsymbol{W}^{(t)}, \tilde{\boldsymbol{\theta}}_{1}^{(t-1}, \ldots, \tilde{\boldsymbol{\theta}}_{L}^{(t-1)}\right)
$$

and further combining (32) and (34), we have

$$
\Gamma^{(t)}\left(\boldsymbol{W}^{(t)}, \tilde{\boldsymbol{\Theta}}^{(t)}\right) \geq \Gamma^{(t-1)}\left(\boldsymbol{W}^{(t-1)}, \tilde{\boldsymbol{\Theta}}^{(t-1)}\right),
$$

for all $t>0$. That is, $\Gamma^{(t)}$ 's is a non-decreasing sequence. It is obvious that due to the limited transmit power, the SINR $\gamma_{k^{(l)}}^{(t)}$ is bounded from the above by its SNR (by ignoring the interference terms); hence, $\Gamma^{(t)}$ is bounded from the above as well. Therefore, Algorithm 1 is guaranteed to converge.

Next, we show that the algorithm converges to a local optimum. The following proof is similar to those in [50], [51]. When Algorithm 1 converges, denote the solution as $\hat{\chi}^{*}=\left\{\boldsymbol{w}_{k^{(l)}}^{*}, \tilde{\boldsymbol{\theta}}_{l}^{*}\right\}$. For each sub-problem (P4.2) shown in (19), if it is feasible, the local optimum is guaranteed to be achieved by the CVXPY/DCCP solver. Let $\left\{\tilde{\boldsymbol{\theta}}_{\hat{l}}^{*}, \boldsymbol{u}^{*}, \boldsymbol{v}^{*}, s^{*}\right\}$ denote the locally optimal solution; then $\boldsymbol{\theta}_{\hat{l}}^{*}=\tilde{\boldsymbol{\theta}}_{\hat{l}}^{*}\left[1: M_{t} / 2\right]+$ $j \tilde{\boldsymbol{\theta}}_{\hat{l}}^{*}\left[M_{t} / 2+1: M_{t}\right]$ is a locally optimal solution of the problem (P4.1) in (14). The Karush-Kuhn-Tucker (KKT) condition for the problem (P4.1) is satisfied at $\boldsymbol{\theta}_{\hat{l}}^{*}$, for each $\hat{l}$. Let $\tilde{\Gamma}\left(\boldsymbol{\theta}_{\hat{l}}\right)$ be the objective function of (P4.1), and $R\left(\hat{\chi}^{*}\right)=$ 
$\left[R_{1}\left(\hat{\chi}^{*}, R_{2}\left(\hat{\chi}^{*}\right)\right), \ldots, R_{I}\left(\hat{\chi}^{*}\right)\right.$ is the set of the constraints of the problem (P4.1). Then, we can write

$$
\begin{aligned}
& \nabla_{\boldsymbol{\theta}_{i}^{*}} \tilde{\Gamma}\left(\hat{\boldsymbol{\chi}}^{*}\right)+Y^{T} \nabla_{\boldsymbol{\theta}_{i}^{*}} R\left(\hat{\boldsymbol{\chi}}^{*}\right)=0 \\
& y_{i} \geq 0, y_{i} R\left(\hat{\boldsymbol{\chi}}^{*}\right)=0, \forall i .
\end{aligned}
$$

where $Y=\left[y_{1}, y_{2}, \ldots, y_{I}\right]$ is the optimal Lagrangian variable set, and $\nabla_{\boldsymbol{x}}$ is partially derivative with respect to $\boldsymbol{x}$. We also note that $\left\{\boldsymbol{w}_{k_{(l)}^{*}}^{*}\right\}$ is a globally optimal solution for (8), hence its KKT condition is also satisfied with respect to $\boldsymbol{W}=\left\{\boldsymbol{w}_{k^{(l)}}^{*}\right\}$, that is

$$
\begin{aligned}
& \nabla_{W} \tilde{\Gamma}\left(\hat{\chi}^{*}\right)+Y^{T} \nabla_{W} R\left(\hat{\chi}^{*}\right)=0 \\
& y_{i} \geq 0, y_{i} R\left(\hat{\chi}^{*}\right)=0, \forall i .
\end{aligned}
$$

Combining all conditions in (36) and (38), we have

$$
\begin{aligned}
& \nabla_{\hat{\chi}^{*}} \tilde{\Gamma}\left(\hat{\chi}^{*}\right)+Y^{T} \nabla_{\hat{\chi}^{*}} R\left(\hat{\chi}^{*}\right)=0 \\
& y_{i} \geq 0, y_{i} R\left(\hat{\chi}^{*}\right)=0, \forall i .
\end{aligned}
$$

which is the KKT condition for (P4.1) in (6), i.e., $\hat{\chi}^{*}$ is a locally optimal solution for (6).

\section{APPENDIX C \\ PROOF OF THEOREM 3}

First, we prove that the sequence $\gamma_{\min }^{(t)}$ is non-decreasing, or $\gamma_{\min }^{(t)} \geq \gamma_{\min }^{(t-1)}$ for all $t>0$. In step 5 of Algorithm 2, we obtain $\boldsymbol{w}_{k^{(l)}}^{(t)}$ from $\left\{\boldsymbol{\theta}_{l}^{(t-1)}\right\}$ by solving (8), which is the optimal MMSE solution. Thus, we have $\gamma_{k^{(l)}}^{(t)}\left(\boldsymbol{w}_{k^{(l)}}^{(t)}, \boldsymbol{\Theta}^{(t-1)}\right) \geq$ $\gamma_{k^{(l)}}^{(t-1)}\left(\boldsymbol{w}_{k^{(l)}}^{(t-1)}, \boldsymbol{\Theta}^{(t-1)}\right)$, for all $l, k$. It follows that the minimum achievable rate among the users satisfies

$$
\gamma_{\min }^{(t)}\left(\boldsymbol{W}^{(t)}, \boldsymbol{\Theta}^{(t-1)}\right) \geq \gamma_{\min }^{(t-1)}\left(\boldsymbol{W}^{(t-1)}, \Theta^{(t-1)}\right) .
$$

Next in step 6 and 7, for each $\hat{l} \in \mathcal{L}$, we obtain $\Psi_{\hat{l}}^{(t)}$ by solving (24) using the CVX solver with the bisection search, which is guaranteed to find the optimal value for this sub-problem. The heuristic check in step 8-14 ensures that

$$
\gamma_{\min }^{(t)}\left(\boldsymbol{\theta}_{\hat{l}}^{(t)}\right) \geq \gamma_{\min }^{(t)}\left(\boldsymbol{\theta}_{\hat{l}}^{(t-1)}\right),
$$

for all $\hat{l} \in \mathcal{L}$. Based on the above, we have

$$
\gamma_{\min }^{(t)}\left(\boldsymbol{W}^{(t)}, \boldsymbol{\Theta}^{(t)}\right) \geq \gamma_{\min }^{(t)}\left(\boldsymbol{W}^{(t)}, \boldsymbol{\Theta}^{(t-1)}\right) .
$$

Combine (39) and (40), we further have

$$
\gamma_{\min }^{(t)}\left(\boldsymbol{W}^{(t)}, \Theta^{(t)}\right) \geq \gamma_{\min }^{(t-1)}\left(\boldsymbol{W}^{(t-1)}, \boldsymbol{\Theta}^{(t-1)}\right),
$$

for all $t>0$. That is, $\gamma_{\min }^{(t)}$ 's is a non-decreasing sequence. Due to the limited transmit power, the SINR $\gamma_{k^{(l)}}^{(t)}$ is bounded from the above by its SNR (by ignoring the interference terms); hence, $\gamma_{\min }^{(t)}$ is bounded from the above as well. Therefore, Algorithm 2 is guaranteed to converge.

\section{REFERENCES}

[1] M. Di Renzo, M. Debbah, Dinh-Thuy Phan-Huy, A. Zappone, M. Alouini, C. Yuen, V. Sciancalepore, G. Alexandropoulos, J. Hoydis, H. Gacanin, J. de Rosny, A. Bounceur, G. Lerosey,and M. Fink, "Smart radio environments empowered by reconfigurable AI meta-surfaces: An idea whose time has come," EURASIP J. Wireless Commun. Netw., vol. 2019:129, May 2019.

[2] M. Di Renzo, A. Zappone, M. Debbah, M. S. Alouini, C. Yuen, J. de Rosny, and S. Tretyakov, "Smart radio environments empowered by reconfigurable intelligent surfaces: How it works, state of research, and the road ahead", IEEE J. Sel. Areas Commun., vol. 38, no. 11, Nov. 2020.

[3] E. Basar, M. Di Renzo, J. De Rosny, M. Debbah, M. S. Alouini, and R. Zhang, "Wireless communications through reconfigurable intelligent surfaces," IEEE Access, pp. 116753-116773, 2019.

[4] Q. Wu, S. Zhang, B. Zheng, C. You, and R. Zhang, "Intelligent reflecting surface-aided wireless communications: A tutorial," IEEE Transactions on Communications, vol. 69, no. 5, pp. 3313-3351, May 2021.

[5] Q. Wu and R. Zhang, "Towards smart and reconfigurable environment: intelligent reflecting surface aided wireless network," IEEE Communications Magazine, vol. 58, no. 1, pp. 106-112, Jan. 2020.

[6] B. Zheng, C. You, and R. Zhang, "Fast channel estimation for IRSassisted OFDM," IEEE Wireless Communications Letters, vol. 10, no. 3, pp. 580-584, Mar. 2021.

[7] Q. Wu and R. Zhang, "Intelligent reflecting surface enhanced wireless network via joint active and passive beamforming," IEEE Trans. Wireless Commun., vol. 18, no. 11, pp. 5394-5409, Nov. 2019.

[8] C. Huang, A. Zappone, G. C. Alexandropoulos, M. Debbah, and C. Yuen, "Reconfigurable intelligent surfaces for energy efficiency in wireless communication," IEEE Trans. Wireless Commun., vol. 18, no. 8, pp. 4157-4170, Aug. 2019.

[9] C. You, B. Zheng, and R. Zhang, "Channel estimation and passive beamforming for intelligent reflecting surface: Discrete phase shift and progressive refinement," IEEE J. Sel. Areas Commun., vol. 38, no. 11, pp. 2604-2620, Nov. 2020.

[10] Q. U. A. Nadeem, A. Kammoun, A. Chaaban, M. Debbah, and M. S. Alouini, "Asymptotic max-min SINR analysis of reconfigurable intelligent surface assisted MISO systems," IEEE Transactions on Wireless Communications, vol. 19, no. 12, pp. 7748-7764, Dec. 2020.

[11] M. Di Renzo et al., "Reconfigurable intelligent surfaces vs. relaying: Differences, similarities, and performance comparison," IEEE Open Journal of the Communications Society, vol. 1, pp. 798-807, 2020.

[12] C. Huang et al., "Holographic MIMO surfaces for 6G wireless networks: Opportunities, challenges, and trends," IEEE Wireless Communications, vol. 27, no. 5, pp. 118-125, October 2020.

[13] Z. Yang, M. Chen, W. Saad, W. Xu, M. Shikh-Bahaei, H. V. Poor, and S. Cui, "Energy-efficient wireless communications with distributed reconfigurable intelligent surfaces," IEEE Transactions on Wireless Communications, Jul. 2021.

[14] X. Li, J. Fang, F. Gao, and H. Li, "Joint active and passive beamforming for intelligent reflecting surface-assisted massive MIMO systems," arXiv preprint arXiv:1912.00728, 2019.

[15] L. Yang, Y. Yang, D. B. da Costa, and I. Trigui, "Outage probability and capacity scaling law of multiple RIS-aided cooperative networks," arXiv preprint arXiv:2007.13293, 2020.

[16] B. Zheng, C. You, and R. Zhang, "Double-IRS assisted multi-user MIMO: Cooperative pPassive beamforming design," IEEE Trans. on Wireless Communications, vol. 20, no. 7, pp. 4513-4526, July 2021.

[17] Y. Han, S. Zhang, L. Duan, and R. Zhang, "Cooperative double-IRS aided communication: Beamforming design and power scaling," IEEE Wireless Communications Letters, vol. 9, no. 8, pp. 1206-1210, Aug. 2020.

[18] Tu V. Nguyen and Diep N. Nguyen, "Secondary Reflections Amongst Multiple IRSs: Friends or Foes?" submitted to the IEEE Wireless Communications and Networking Conference, Texas Austin, 2022 (under review).

[19] E. Bjornson, O. Ozdogan, and E. G. Larsson, "Intelligent reflecting surface versus decode-and-forward: How large surfaces are needed to beat relaying?" IEEE Wireless Commun. Lett., vol. 9, no. 2, pp. 244-248, Feb. 2020

[20] C. You, Z. Kang, Y. Zeng, and R. Zhang, "Enabling smart reflection in integrated air-ground wireless network: IRS meets UAV," arXiv preprint arXiv:2103.07151, Mar. 2021. 
[21] B. Zheng and R. Zhang. "Intelligent reflecting surface-enhanced OFDM: Channel estimation and reflection optimization," IEEE Wireless Communications Letters, pp. 518-522, 2020.

[22] D. P. Bertsekas, Nonlinear Programming. Belmont, MA, USA: Athena Scientific, 2nd edition, 1999.

[23] Steven M. Kay. Fundamentals of Statistical Signal Processing: Estimation Theory, Pearson, 1st edition, 2017.

[24] S. Boyd and L. Vandenberghe, Convex Optimization, Cambridge University Press, 1st edition, 2004.

[25] M. Grant and S. Boyd, "CVX: Matlab software for disciplined convex programming," 2016. [Online]. Available: http://cvxr.com/cvx

[26] S. Diamond and S. Boyd, "CVXPY: A Python-embedded modeling language for convex optimization," The Journal of Machine Learning Research, Volume 17, Issue 1, Jan 2016, pp 2909-2913.

[27] X. Shen, S. Diamond, Y. Gu, and S. Boyd, "Disciplined convexconcave programming," in 2016 IEEE 55th Conference on Decision and Control $(C D C)$, 2016. A full version available online: stanford.edu/ boyd/papers/pdf/dccp.pdf

[28] Z. Luo, W. Ma, A. M. So, Y. Ye, and S. Zhang, "Semidefinite relaxation of quadratic optimization problems," IEEE Signal Process. Mag., vol. 27, no. 3, pp. 20-34, May 2010.

[29] S. S. Christensen, R. Agarwal, E. De Carvalho, and J. M. Cioffi, "Weighted sum-rate maximization using weighted MMSE for MIMOBC beamforming design," IEEE Transactions on Wireless Communications, vol. 7, no. 12, pp. 4792-4799, December 2008.

[30] Q. Shi, M. Razaviyayn, Z. Luo, and C. He, "An iteratively weighted MMSE approach to distributed sum-utility maximization for a MIMO interfering broadcast channel," IEEE Transactions on Signal Processing, vol. 59, no. 9, pp. 4331-4340, Sept. 2011

[31] L. Liu, R. Zhang, and K. Chua, "Achieving global optimality for weighted sum-rate maximization in the K-user Gaussian interference channel with multiple antennas," IEEE Transactions on Wireless Communications, vol. 11, no. 5, pp. 1933-1945, May 2012.

[32] A. C. Cirik, R. Wang, Y. Hua, and M. Latva-aho, "Weighted sumrate maximization for full-duplex MIMO interference channels," IEEE Transactions on Communications, vol. 63, no. 3, pp. 801-815, March 2015.

[33] P. Aquilina, A. C. Cirik, and T. Ratnarajah, "Sum rate maximization in full-duplex multi-user multi-cell MIMO networks," IEEE Transactions on Communications, vol. 65, no. 4, pp. 1590-1608, April 2017.

[34] Z. Li, M. Hua, Q. Wang, and Q. Song, "Weighted sum-rate maximization for multi-RS aided cooperative transmission," IEEE Wireless Communications Letters, vol. 9, no. 10, pp. 1620-1624, Oct. 2020.

[35] M. A. Saeidi, M. J. Emadi, H. Masoumi, M. R. Mili, D. W. K. Ng, and I. Krikidis, "Weighted sum-rate maximization for multi-IRS-assisted full-duplex systems with hardware impairments," IEEE Transactions on Cognitive Communications and Networking, vol. 7, no. 2, pp. 466481, June 2021.

[36] D. Gesbert, S. Hanly, H. Huang, S. S. Shamai, S. Osvaldo, and W. Yu, "Multi-cell MIMO cooperative networks: A new look at interference," IEEE Journal on Selected Areas in Communications vol. 28, no. 9, pp. 1380-1408, 2010.

[37] H. Guo, Y. Liang, J. Chen, and E. G. Larsson, "Weighted sumrate maximization for reconfigurable intelligent surface aided wireless networks," IEEE Transactions on Wireless Communications, vol. 19, no. 5, pp. 3064-3076, May 2020.

[38] C. Pan, H. Ren, K. Wang, W. Xu, M. Elkashlan, A. Nallanathan, and L. Hanzo, "Multicell MIMO communications relying on intelligent reflecting surfaces," IEEE Transactions on Wireless Communications, vol. 19, no. 8, pp. 5218-5233, Aug. 2020.

[39] W. Mei and R. Zhang, "Performance analysis and user association optimization for wireless network aided by multiple intelligent reflecting surfaces," IEEE Transactions on Communications, Jun. 2021.

[40] Q. Wu and R. Zhang, "Joint active and passive beamforming optimization for intelligent reflecting surface assisted SWIPT under QoS constraints," IEEE Journal on Selected Areas in Communications, vol. 38, no. 8, pp. 1735-1748, Aug. 2020.

[41] C. Cai, X. Yuan, W. Yan, Z. Huang, Y. -C. Liang, and W. Zhang, "Hierarchical passive beamforming for reconfigurable intelligent surface aided communications," IEEE Wireless Communications Letters, Jun. 2021.

[42] W. Yan, X. Yuan, Z. -Q. He, and X. Kuai, "Passive beamforming and information transfer design for reconfigurable intelligent surfaces aided multiuser MIMO systems," IEEE Journal on Selected Areas in Communications, vol. 38, no. 8, pp. 1793-1808, Aug. 2020.

[43] A. Abrardo, D. Dardari, and M. Di Renzo, "Intelligent reflecting surfaces: Sum-rate optimization based on statistical position information,"
IEEE Transactions on Communications, vol. 69, no. 10, pp. 7121-7136, Oct. 2021.

[44] A. Abrardo, D. Dardari, M. Di Renzo, and X. Qian, "MIMO interference channels assisted by reconfigurable intelligent surfaces: Mutual coupling aware sum-rate optimization based on a mutual impedance channel model", arXiv preprint arXiv:2102.07155, 2021.

[45] N. S. Perović, L. -N. Tran, M. Di Renzo, and M. F. Flanagan, "On the maximum achievable sum-rate of the ris-aided mimo broadcast channel," arXiv preprint arXiv:2110.01700, 2021.

[46] W. Mei and R. Zhang, "Multi-beam multi-hop routing for intelligent reflecting surfaces aided massive MIMO," arXiv preprint arXiv:2101.00217, 2021.

[47] W. Mei, B. Zheng, C. You, and R. Zhang, "Intelligent reflecting surface aided wireless networks: From single-reflection to multi-reflection design and optimization", arXiv preprint arXiv:2109.13641, 2021.

[48] W. Mei and R. Zhang, "Distributed beam training for intelligent reflecting surface enabled multi-hop routing," IEEE Wireless Commun. Lett., 2021, early access.

[49] C. Huang, Z. Yang, G. C. Alexandropoulos, K. Xiong, L. Wei, C. Yuen, Z. Zhang, and M. Debbah, "Multi-hop RIS-empowered terahertz communications: A DRL-based hybrid beamforming design," IEEE J. Sel. Areas Commun., vol. 39, no. 6, pp. 1663-1677, Jun. 2021.

[50] N. T. Nguyen, D. N. Nguyen, D. T. Hoang, N. Van Huynh, E. Dutkiewicz, N. H. Nguyen, and Q. T. Nguyen, "Time scheduling and energy trading for heterogeneous wireless-powered and backscatteringbased IoT networks," IEEE Transactions on Wireless Communications, vol. 20, no. 10, pp. 6835-6851, Oct. 2021.

[51] D. Feng, G. Yu, C. Xiong, Y. Yuan-Wu, G. Y. Li, G. Feng, and S. Li, "Mode switching for energy-efficient device-to-device communications in cellular networks," IEEE Transactions on Wireless Communications, vol. 14 , no. 12 , pp. $6993-7003$, Dec. 2015. 